\title{
Imposing Mixed Dirichlet-Neumann-Robin Boundary Conditions in a Level-Set Framework
}

\author{
Ásdís Helgadóttir * $\quad$ Yen Ting $\mathrm{Ng}^{\dagger} \quad$ Chohong Min ${ }^{\ddagger} \quad$ Frédéric Gibou ${ }^{\dagger \S}$
}

May 6, 2015

\begin{abstract}
We consider the Poisson equation with mixed Dirichlet, Neumann and Robin boundary conditions on irregular domains. We describe a straightforward and efficient approach for imposing the mixed boundary conditions using an hybrid finite-volume/finite-difference approach, leveraging on the work of $[14,30,33]$. We utilize three different level set functions to represent the irregular boundary at which each of the three different boundary conditions must be imposed; as a consequence, this approach can be applied to moving boundaries. The method is straightforward to implement, produces a symmetric positive definite linear system and second-order accurate solutions in the $L^{\infty}$-norm in two and three spatial dimensions. Numerical examples illustrate the second-order accuracy and the robustness of the method.
\end{abstract}

\section{Introduction}

The Poisson equation is one of the building blocks in partial-differential-equation based modeling of physical phenomena and has countless applications in fluid dynamics, heat transfer, electrostatics, wave phenomena and a range of other important engineering problems. Many different approaches

*Faculty of Industrial Engineering, Mechanical Engineering and Computer Science Computer Science Department, University of Iceland, Reykjavik, 107 Iceland, asdishe@hi.is +354 5254917.

${ }^{\dagger}$ Computer Science Department, University of California, Santa Barbara, CA 93106.

${ }^{\ddagger}$ Mathematics Department and Research Institute for Basic Sciences, KyungHee University, Seoul, Korea 130-701

$\S$ Mechanical Engineering Department, University of California, Santa Barbara, CA 93106

(C) 2015. This manuscript version is made available under the Elsevier user license http://www.elsevier.com/open-access/userlicense/1.0/ 
have been proposed for solving the Poisson problem subjected to different boundary conditions. The main methods used to solve the Poisson equation are finite element (e.g. [1, 4, 24, 9, 18, 17, $42,8,5,15,34]$ and the references therein) or finite difference/volume methods (e.g. [26, 44, 2, 45, $27,14,12,22,6,46]$ and the references therein).

The advantage of a finite element approach is that symmetric positive definite linear systems are always constructed and a posteriori error estimates can be used to construct mesh refinement criteria that minimize the overall error. Analysis of finite element schemes and order of accuracy of the methods are also possible using norms induced by the solution's space. The main drawback of finite element methods in arbitrary geometry is the difficulty associated with the computational complexity of the mesh generation. This comes from the fact that the elements must conform to the irregular domains' boundary and skewed elements can corrupt the accuracy of the method. This leads to a significant computational burden, especially in the case where frequent refinement is necessary, as it is the case in free boundary problems.

Various methods have been used to enforce the correct boundary conditions at an irregular interface. The immerse boundary method smears out the interface condition using a $\delta$-function formulation, leading to a simple numerical scheme. A drawback is the loss of accuracy near the boundary (see [35, 36, 37, 38, 43]). The immerse interface method (IIM) [25] is second-order accurate method, including near the interface, but it is more difficult to implement, especially in three spatial dimensions. IIM produces sparse but neither symmetric nor positive definite linear systems, which are more costly to solve than symmetric positive definite versions. The immerse interface method seeks to minimize the truncation error of static two dimensional problems and is not a robust secondorder accurate method $[27,20]$. Liu et al. presented a method for discretizing the variable coefficient Poisson equation where the solution and its derivatives may have jumps across the interface in [27]. This discretization is particularly important in applications such as two-phase incompressible flow and flame simulations (see e.g. [23, 31, 11]). This method is straightforward to implement since only the right-hand-side of the linear system is modified, hence preserving the standard symmetric definite positive (SPD) discretization of the Poisson equation on regular domains. The solutions are first-order accurate in the $L^{\infty}$-norm. Second order accurate solutions to the Poisson equation with jumps across interfaces have for example been developed in [32, 21]. In those methods, the 
stencil of the matrix is however much greater and the matrix generally not symmetric. Recently, Guittet et al. introduce the Voronoi interface method (VIM) [16]. This method construct a band Voronoi cells near the interface and applies the idea of the ghost fluid method of Liu et al. [23] on those cells, whose faces are orthogonal to the fluxes. As a consequence only the right-hand-side of the solver is modified and the techniques produces second-order accurate solutions and symmetric positive definite linear systems.

Gibou et al. proposed a method for imposing Dirichlet boundary conditions instead of jump conditions in [14], with applications to free surface flows and diffusion dominated moving boundary problems (see e.g. $[28,10,13])$. This method is straightforward to implement, produces an SPD linear system and second-order accurate solutions in the $L^{\infty}$-norm. In addition, it has been extended to fourth-order accuracy, albeit non-symmetric, in [12]. Finite volume approaches allow Neumann and Robin boundary conditions to be treated in a straight forward manner leading to the development of hybrid finite volume / level set methods in [30, 33, 19]. In particular, Papac et al. [33] describes a simple method for the case of Robin boundary conditions that produces second-order accurate solutions in the $L^{\infty}$-norm and a SPD linear system. Purvis et al. [39] and later Ng et al. [30] presented a second-order accurate SPD method for imposing Neumann boundary conditions on irregular domains in the context of fluid flows in arbitrary shaped solid objects. This is an advantage over the more complicated method of Jomaa et al. [22], for which non-symmetric linear systems are obtained, although the accuracy of the gradients may drop to first-order. Bedrossian et al. presented an approach for imposing jump conditions in the solution and solution's gradients on irregular domains in [3] and applied this framework to the case of Dirichlet and Neumann boundary conditions as well so this method can be applied to mixed boundary conditions. The linear systems are SPD and the solutions are second-order accurate but the method is not straightforward to implement. In [7] Coco et al. present a finite difference ghost-cell multigrid approach for the Poisson equation with mixed Neumann and Dirichlet boundary conditions on arbitrary domains. There the Neumann boundary condition is always a smooth extension of the Dirichlet boundary conditions and vise versa (i.e. there is never a kink in the irregular interface where the two boundary conditions meet even though there may be a kink in the interface). The method is second order accurate so is its gradient. So far, no test are shown in spatial three dimensions. None of the 
previously mentioned solvers have, therefore, shown that they handle three dimensional examples where mixture of all three types of boundary conditions on the irregular interface where kinks can occur where the boundary conditions meet.

In this paper, we focus on the Poisson problem with mixed Dirichlet-Neumann-Robin boundary conditions. Such boundary conditions can be encountered for example in the simulation of free surface flows on an arbitrarily shaped topography (Dirichlet-Neumann) or the simulation of heat diffusion under convection cooling on part of the computational domain (Robin-Dirichlet or RobinNeumann). We describe an approach for imposing mixed Dirichlet and/or Neumann and/or Robin boundary conditions in a straightforward and robust fashion, based on combining and extending some of our prior work into a unified framework. This method is unconditionally stable, produces a SPD linear system and second-order accurate solutions in the $L^{\infty}$-norm but its gradient is first order accurate.

\section{Equations and Numerical Method}

We considered the Poisson problem on a domain $\Omega$ separated into two disjoint subsets $\Omega^{-}$and $\Omega^{+}$ such that $\Omega=\Omega^{-} \cup \Omega^{+}$, and $\Gamma$ is the interface between $\Omega^{-}$and $\Omega^{+}$. We employ three implicit functions, $\phi^{D}, \phi^{N}$ and $\phi^{R}$ to describe the different regions where the solution $u$ is computed as well as where the different boundary conditions are imposed (see figure 1). In particular, we are interested in solving the Poisson equation only inside $\Omega^{-}=\max \left\{\phi^{D}, \phi^{N}, \phi^{R}\right\}$. Dirichlet, Neumann and Robin boundary conditions are applied on $\Gamma=\left\{\phi^{D}=0 \wedge \phi^{N}<0 \wedge \phi^{R}<0\right\} \cap\left\{\phi^{N}=0 \wedge \phi^{D}<\right.$ $\left.0 \wedge \phi^{R}<0\right\} \cap\left\{\phi^{R}=0 \wedge \phi^{D}<0 \wedge \phi^{N}<0\right\}$, respectively. Mathematically, the problem is described as solving for the solution $u$ at a location $\mathbf{x}$ satisfying:

$$
\begin{array}{rlrl}
\Delta u & =F & \mathbf{x} \in \Omega^{-}, \\
u & =G & & \text { on } \phi^{D}=0, \\
\frac{\partial u}{\partial n} & =K & & \text { on } \phi^{N}=0, \\
\frac{\partial u}{\partial n}+\alpha u & =M & & \text { on } \phi^{R}=0,
\end{array}
$$

where $\alpha>0$. 


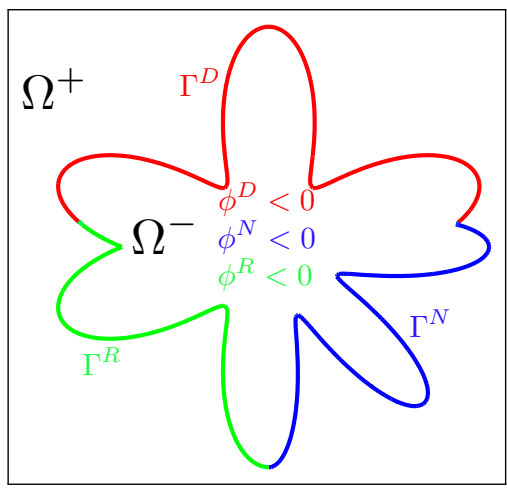

(a) A computational domain in $2 \mathrm{D}$.

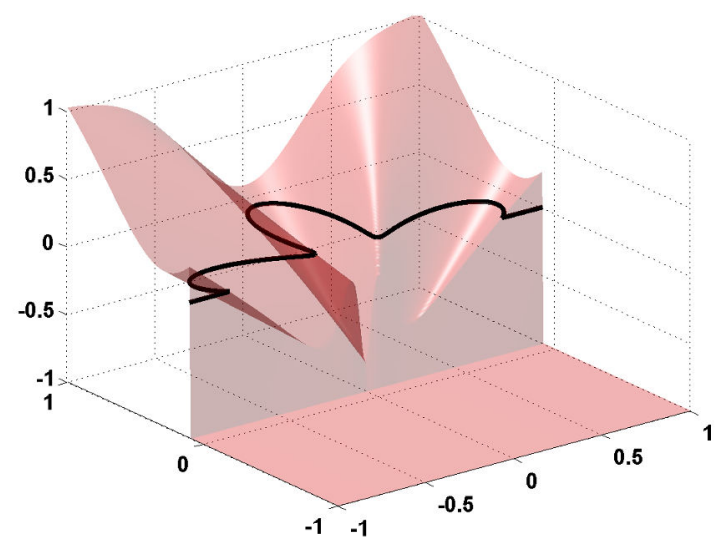

(b) Surface plot (red) and 0-level set (black) of $\phi^{D}$.

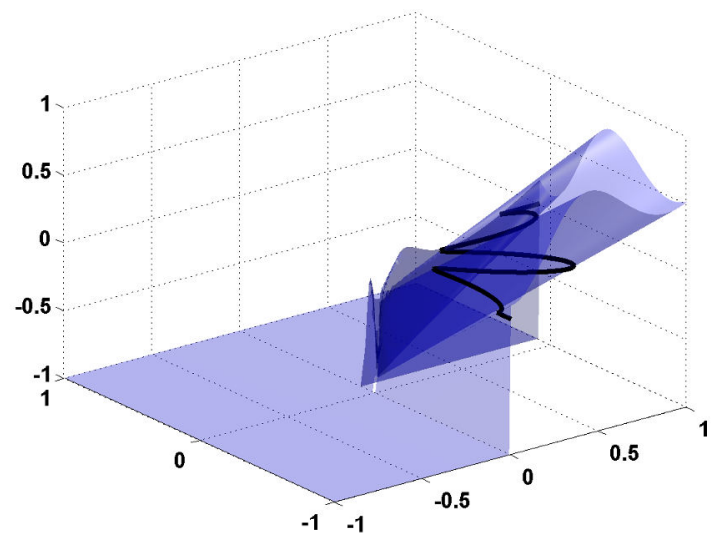

(c) Surface plot (blue) and 0-level set (black) of $\phi^{N}$.

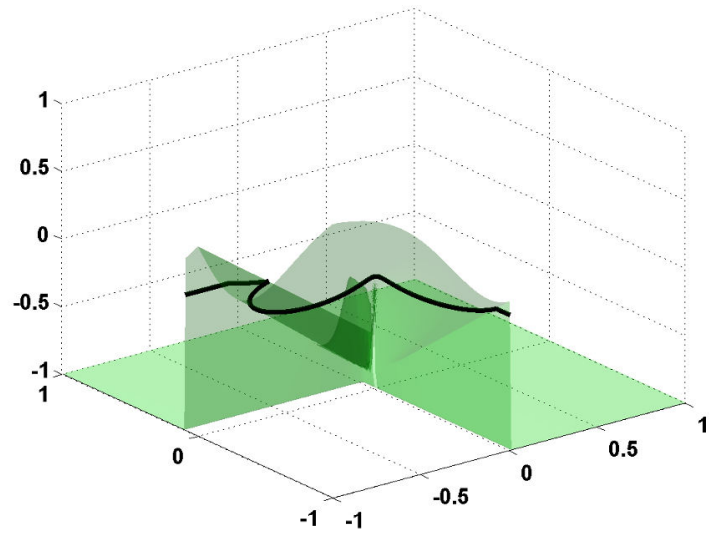

(d) Surface plot (green) and 0-level set (black) of $\phi^{R}$.

Figure 1: A two-dimensional computational domain and its representation. The solution is computed in $\Omega^{-}$and the Dirichlet, Neumann and Robin boundary conditions are applied on $\Gamma^{D}, \Gamma^{N}$ and $\Gamma^{R}$, respectively. The level-set functions $\phi^{D}, \phi^{N}$ and $\phi^{R}$ have been set arbitrarily to 1 in $\Omega^{+}$for visualization purposes; in practice these functions are Lipschitz continuous. 
We consider a finite volume discretization for imposing the Neumann and Robin boundary conditions, as in [30, 33, 40]: Consider a cell $C_{i j}=\left[i-\frac{1}{2}, i+\frac{1}{2}\right] \times\left[j-\frac{1}{2}, j+\frac{1}{2}\right]$ partially covered by the irregular domain $\Omega^{-}$. Taking a finite volume approach, i.e. integrating the left hand side of equation (1) over $C_{i j}$ and evoking the divergence theorem, we obtain:

$$
\int_{C_{i j} \cap \Omega^{-}} \nabla \cdot \nabla u d \Omega=\int_{\partial\left(C_{i j} \cap \Omega^{-}\right)} n \cdot \nabla u d \Gamma
$$

where $d \Omega$ and $d \Gamma$ refer to the area and length differentials respectively, in two spatial dimensions. Since the boundary $\partial\left(C_{i j} \cap \Omega^{-}\right)$has two components, the faces of the grid cell $\partial C_{i j} \cap \Omega^{-}$and the interface with the irregular external boundary $C_{i j} \cap \Gamma$, we consider separately the contribution of the two components:

$\int_{\partial\left(C_{i j} \cap \Omega^{-}\right)} n \cdot \nabla u d \Gamma \approx \int_{\partial C_{i j} \cap \Omega^{-}} n \cdot \nabla u d \Gamma+\int_{C_{i j} \cap \Gamma^{N}} K d \Gamma+\int_{C_{i j} \cap \Gamma^{R}} M d \Gamma-\alpha u_{i, j} \int_{C_{i j} \cap \Gamma^{R}} d \Gamma$.

By approximating the boundary integral on the grid faces as the product of the length and the sampled value at the center, we obtain:

$$
\begin{aligned}
\int_{\partial\left(C_{i j} \cap \Omega^{-}\right)} n \cdot \nabla u d \Gamma & \simeq L_{i+\frac{1}{2}, j} \frac{u_{i+1, j}-u_{i, j}}{\Delta x} \\
& -L_{i-\frac{1}{2}, j} \frac{u_{i, j}-u_{i-1, j}}{\Delta x} \\
& +L_{i, j+\frac{1}{2}} \frac{u_{i, j+1}-u_{i, j}}{\Delta y} \\
& -L_{i, j-\frac{1}{2}} \frac{u_{i, j}-u_{i, j-1}}{\Delta y} \\
& -\alpha u_{i, j} \int_{C_{i j} \cap \Gamma^{R}} d \Gamma+\int_{C_{i j} \cap \Gamma^{N}} K d \Gamma+\int_{C_{i j} \cap \Gamma^{R}} M d \Gamma,
\end{aligned}
$$

where on a face $\left(i-\frac{1}{2}\right) \times\left[j-\frac{1}{2}, j+\frac{1}{2}\right]$, the length fraction $L_{i-\frac{1}{2}, j}$ of the face covered by the irregular domain $\{x \mid \phi(x) \leq 0\}$ is linearly approximated as:

$$
L_{i-\frac{1}{2}, j}= \begin{cases}\Delta y \frac{\phi_{i-\frac{1}{2}, j-\frac{1}{2}}}{\phi_{i-\frac{1}{2}, j-\frac{1}{2}}-\phi_{i-\frac{1}{2}, j+\frac{1}{2}}} & \text { if } \phi_{i-\frac{1}{2}, j-\frac{1}{2}}<0 \text { and } \phi_{i-\frac{1}{2}, j+\frac{1}{2}}>0, \\ \Delta y \frac{\phi_{i-\frac{1}{2}, j+\frac{1}{2}}}{\phi_{i-\frac{1}{2}, j+\frac{1}{2}-\phi_{i-\frac{1}{2}, j-\frac{1}{2}}}} & \text { if } \phi_{i-\frac{1}{2}, j-\frac{1}{2}}>0 \text { and } \phi_{i-\frac{1}{2}, j+\frac{1}{2}}<0, \\ \Delta y & \text { if } \phi_{i-\frac{1}{2}, j-\frac{1}{2}}<0 \text { and } \phi_{i-\frac{1}{2}, j+\frac{1}{2}}<0, \\ 0 & \text { if } \phi_{i-\frac{1}{2}, j-\frac{1}{2}}>0 \text { and } \phi_{i-\frac{1}{2}, j+\frac{1}{2}}>0 .\end{cases}
$$


We, therefore, obtain a linear system for which each row represents the following equation:

$$
\begin{aligned}
& L_{i+\frac{1}{2}, j} \frac{u_{i+1, j}-u_{i, j}}{\Delta x}-L_{i-\frac{1}{2}, j} \frac{u_{i, j}-u_{i-1, j}}{\Delta x} \\
& +L_{i, j+\frac{1}{2}} \frac{u_{i, j+1}-u_{i, j}}{\Delta y}-L_{i, j-\frac{1}{2}} \frac{u_{i, j}-u_{i, j-1}}{\Delta y}-\alpha u_{i, j} \int_{C_{i j} \cap \Gamma^{R}} d \Gamma \\
& \approx \int_{C_{i j} \cap \Omega^{-}} F d \Omega-\int_{C_{i j} \cap \Gamma^{N}} K d \Gamma-\int_{C_{i j} \cap \Gamma^{R}} M d \Gamma .
\end{aligned}
$$

The integrals are found by geometric integration and will be detailed in section 2.1. In particular, in the case where mixed Neumann and Robin boundary conditions are present, a subcell integration of each of the interfaces is crucial for convergence as detailed in section 2.1.

We impose Dirichlet boundary conditions by modifying equation (3) at grid nodes adjacent to the interface $\Gamma^{D}=\left\{\phi^{D}=0\right\}$ using the approach introduced by Gibou et al. [14]: Consider a case where the interface defined by $\phi^{D}=0$ crosses in between grid nodes $x_{i}$ and $x_{i+1}$ (see figure 2), then equation (3) is modified to incorporate the value of $G_{\Gamma}$ at the interface, i.e. the expression:

$$
L_{i+\frac{1}{2}, j} \frac{u_{i+1, j}-u_{i, j}}{\Delta x}-L_{i-\frac{1}{2}, j} \frac{u_{i, j}-u_{i-1, j}}{\Delta x},
$$

is replaced by

$$
L_{i+\frac{1}{2}, j} \frac{G_{\Gamma}-u_{i, j}}{\Delta x_{\Gamma}}-L_{i-\frac{1}{2}, j} \frac{u_{i, j}-u_{i-1, j}}{\Delta x}
$$

where

$$
\begin{aligned}
G_{\Gamma} & =\frac{G_{i+1}\left|\phi_{i}^{D}\right|+G_{i}\left|\phi_{i+1}^{D}\right|}{\left|\phi_{i}^{D}\right|+\left|\phi_{i+1}^{D}\right|}, \\
\Delta x_{\Gamma} & =\Delta x \frac{\phi_{i}^{D}}{\left|\phi_{i}^{D}\right|+\left|\phi_{i+1}^{D}\right|} .
\end{aligned}
$$

Equation (3), modified by equation (4) for grid nodes adjacent to a Dirichlet boundary interface, produces a linear system that enforces mixed Dirichlet, Neumann and Robin boundary conditions at irregular interfaces.

\section{Remarks:}

- It is straightforward to implement such a scheme on arbitrary irregular domains in two and three spatial dimensions and to see that the corresponding linear systems are symmetric positive definite. We are using an incomplete Cholesky preconditioned conjugate gradient method [41] to solve the linear system. 


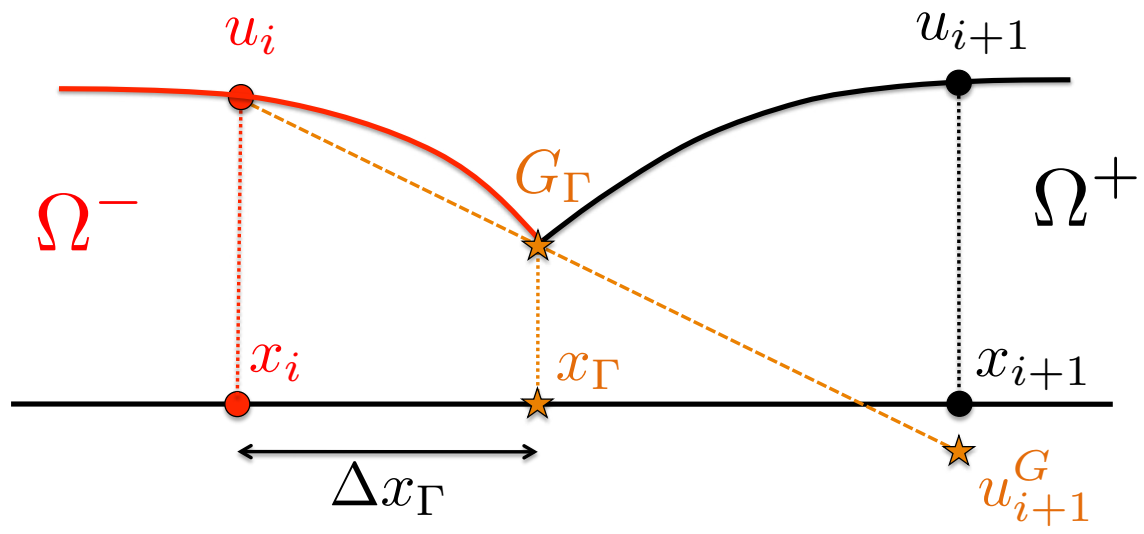

Figure 2: Treatment of Dirichlet boundary conditions on irregular domains. The given interface value $G_{\Gamma}$ is enforced at the interface $\Gamma$ using the approach of [14].

- In the case where a Dirichlet boundary condition is to be imposed in a computational cell along with a Neumann or a Robin boundary condition, we only perform the treatment for the Dirichlet boundary condition, in essence ignoring the Neumann and/or Robin boundary conditions altogether. The method is still clearly second-order accurate. Only in the case where Robin and Neumann boundary conditions are to be imposed in a computational cell, do we calculate the portion of the interface with each of them to account for the correct flux.

\subsection{Geometric Integration}

In order to compute the different integrals in equation (3), we use a modified version of the secondorder accurate geometric integration introduced in [29]: Integrations are performed by first splitting cells $C_{i, j}$ (in two spatial dimensions and $C_{i, j, k}$ in three spatial dimensions) into simplices, $\mathrm{S}$ (i.e. triangles in two spatial dimensions and tetrahedrons in three spatial dimensions). If the sets $S \cap \Gamma$ or $S \bigcap \Omega$ are not simplices they are further split into simplices using a linear interpolation of $\phi$ from the vertices of S as described in [29]. The interface's length inside a simplex or the area of a simplex in two spatial dimensions can be easily found using basic formulas. It is also straightforward to compute the surface of interface inside a simplex or the volume of a simplex in three spatial 

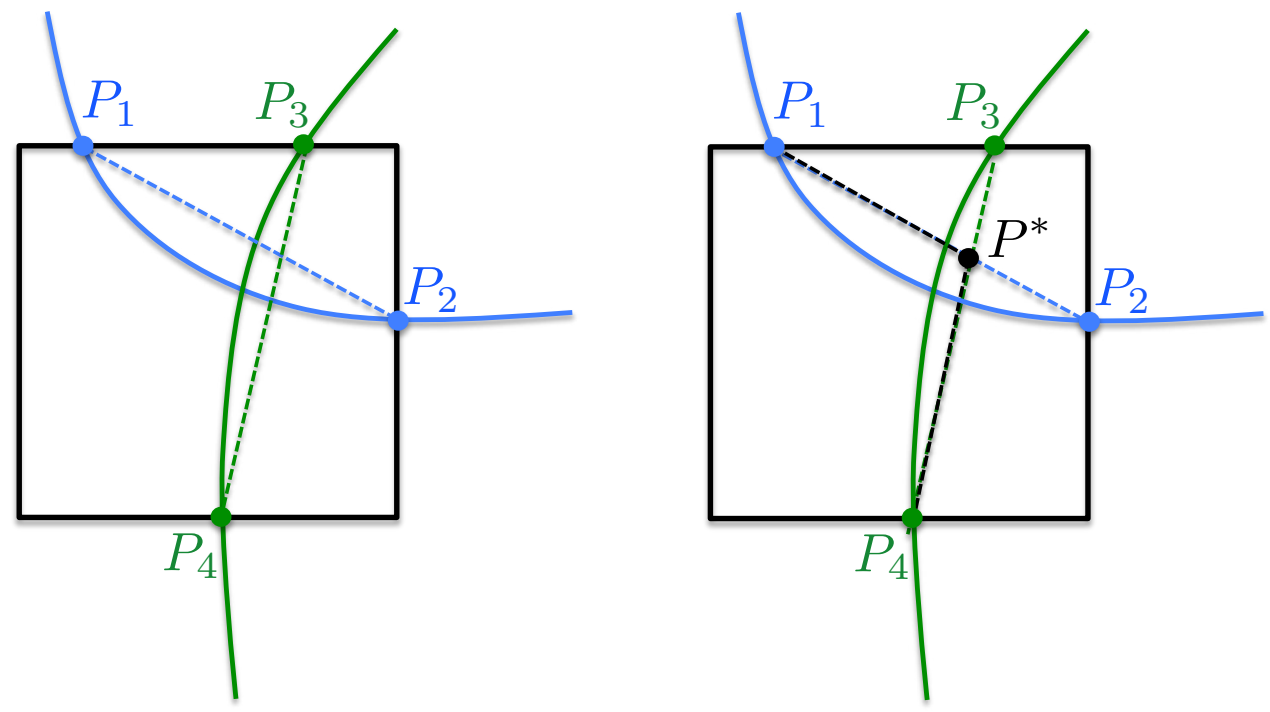

Figure 3: The left schematic depicts the original integration scheme of [29] over both $\Gamma^{N}$ and $\Gamma^{R}$. In this case, the contribution of both Neumann and Robin boundary conditions are overestimated. The schematic on the right depicts the subcell integration described in section 2.1.1. In this case $\Gamma^{N}$ and $\Gamma^{R}$ are correctly only integrated to the cross section point $P^{*}$.

dimensions. Finally, the total integrals are found by adding each integral over all simplices, i.e.

$$
\int_{C_{i, j} \cap \Gamma} f d \Gamma=\sum_{S \in T\left(C_{i, j}\right)} \int_{S \cap \Gamma} f d \Gamma,
$$

and

$$
\int_{C_{i, j} \cap \Omega^{-}} f d \Omega^{-}=\sum_{S \in T\left(C_{i, j}\right)} \int_{S \cap \Omega^{-}} f d \Omega^{-},
$$

where $T\left(C_{i, j}\right)$ represents the triangulation of the current cell and $f$ the function to be integrated, i.e. either $K$ or $M$.

\subsubsection{Subcell Integration}

The integration method described in the previous section involves a single level set function [29]. When both Neumann and Robin boundary conditions are present, then subcell integration is needed 
to avoid a drop in accuracy to first-order. The subcell integration method chosen in this paper is described is this section:

The integration procedure over a domain is straightforward since we can simply follow the procedure described above, except that $\Omega^{-}$is described by $\phi=\max \left(\phi^{N}, \phi^{R}\right)$.

To describe the case of integration over the interface, let's assume that a cell is cut by both $\phi^{N}$ and $\phi^{R}$, as illustrated in figure 3. The points $P_{1}$ and $P_{2}$ intersecting $\phi^{N}$ with the cell's boundary are found, defining a linear approximation of $\phi^{N}$ in that cell. Likewise, we find the points $P_{3}$ and $P_{4}$ on the cell's boundary defining a linear approximation of $\phi^{R}$ in that cell. Then, the intersection point $P^{*}$ between these two linear approximations is used to compute the contribution of $\int_{C_{i, j}} \cap \Gamma^{N}{ }^{N} d \Gamma$ and $\int_{C_{i, j} \cap \Gamma^{R}} M d \Gamma$ in the cell. Specifically, we use:

$\int_{C_{i, j} \cap \Gamma^{N}} K d \Gamma \approx \frac{K\left(P_{1}\right)+K\left(P^{*}\right)}{2} \overline{P_{1} P^{*}} \quad$ and $\quad \int_{C_{i, j} \cap \Gamma^{R}} M d \Gamma \approx \frac{M\left(P_{4}\right)+M\left(P^{*}\right)}{2} \overline{P_{4} P^{*}}$, where $\overline{P_{1} P^{*}}$ and $\overline{P_{4} P^{*}}$ are, respectively, the lengths of the interval between the points $P_{1}, P^{*}$ and $P_{4}, P^{*}$.

\section{Remarks:}

- Special care is needed in the case where both $\phi^{N}$ and $\phi^{R}$ cut through a cell, but do not intersect. In this case, the contribution of each integral is computed separately.

- In three spatial dimensions, the procedure is similar except that planes instead of lines are used as linear approximations.

\section{$3 \quad$ Numerical Experiments}

We present numerical evidence that the proposed method is second-order accurate in both two and three spatial dimensions. 


\subsection{Two Spatial Dimensions}

\subsubsection{Mixed Dirichlet and non-homogenous Neumann boundary conditions}

Consider the Poisson equation on the domain $\Omega=[-1,1] \times[-1,1]$. We define $r=\sqrt{x^{2}+y^{2}}$, $\phi^{D}=-x+.1, \phi^{N}=r-.8, \phi^{R}=-1$, and the exact solution $G=(r-.5)^{3}$ for all $x, y \in \Omega$. Figure 4 depicts the solution and highlights different parts of the interface where Dirichlet and nonhomogenous Neumann boundary conditions are enforced. Table 1 demonstrates the second-order accuracy of the method in the $L^{\infty}$-norm.

\begin{tabular}{|c|c|c|}
\hline Resolution & $\left\|u-u_{h}\right\|_{\infty}$ & Order \\
\hline $32^{2}$ & $1.67 \times 10^{-3}$ & - \\
\hline $64^{2}$ & $4.24 \times 10^{-4}$ & 1.98 \\
\hline $128^{2}$ & $1.18 \times 10^{-4}$ & 1.84 \\
\hline $256^{2}$ & $2.89 \times 10^{-5}$ & 2.03 \\
\hline $512^{2}$ & $7.23 \times 10^{-6}$ & 2.00 \\
\hline $1024^{2}$ & $1.80 \times 10^{-6}$ & 2.01 \\
\hline $2048^{2}$ & $4.59 \times 10^{-7}$ & 1.97 \\
\hline
\end{tabular}

Table 1: Maximum error and rate of maximum error for different resolution for Ex. 3.1.1: Mixed Dirichlet and non-homogenous Neumann boundary conditions on smooth interfaces in two spatial dimensions.

\subsubsection{Mixed Dirichlet and non-homogenous Robin boundary conditions}

Consider the Poisson equation on the domain $\Omega=[-1,1] \times[-1,1]$. We define $r=\sqrt{x^{2}+y^{2}}$, $\phi^{D}=-x+.1, \phi^{N}=-1, \phi^{R}=r-.75$, and the exact solution $G=\exp (x \cdot y)$ for all $x, y \in \Omega$. Figure 5 depicts the solution and highlights different parts of the interface where Dirichlet and non-homogenous Robin boundary conditions are enforced. Table 2 demonstrates the second-order accuracy of the method in the $L^{\infty}$-norm. 


\begin{tabular}{|c|c|c|}
\hline Resolution & $\left\|u-u_{h}\right\|_{\infty}$ & Order \\
\hline $16^{2}$ & $4.87 \times 10^{-3}$ & - \\
\hline $32^{2}$ & $1.12 \times 10^{-3}$ & 2.11 \\
\hline $64^{2}$ & $3.15 \times 10^{-4}$ & 1.83 \\
\hline $128^{2}$ & $8.05 \times 10^{-5}$ & 1.97 \\
\hline $256^{2}$ & $2.14 \times 10^{-5}$ & 1.91 \\
\hline $512^{2}$ & $5.39 \times 10^{-6}$ & 1.99 \\
\hline $1024^{2}$ & $1.38 \times 10^{-6}$ & 1.97 \\
\hline $2048^{2}$ & $3.45 \times 10^{-7}$ & 2.00 \\
\hline
\end{tabular}

Table 2: Maximum error and rate of maximum error for different resolution for Ex. 3.1.2: Mixed Dirichlet and non-homogenous Robin boundary conditions on smooth interfaces in two spatial dimensions.

\subsubsection{Mixed Dirichlet and non-homogenous Neumann boundary conditions}

Consider the Poisson equation on the domain $\Omega=[-1,1] \times[-1,1]$. We define the following for $x, y \in \Omega:$

$$
\begin{aligned}
r & =\sqrt{x^{2}+y^{2}} \\
\theta & =\tan ^{-1}\left(\frac{y}{x}\right) \\
\phi^{D} & = \begin{cases}-\min (r-0.1,0.6+0.3 \cos (6 \theta)-r) & \text { if } x \geq 0 \\
-\min \left(r-0.1,1.1-\sqrt{(x+1.1 \sqrt{0.91})^{2}+y^{2}}\right. & \text { if } x<0\end{cases} \\
\phi^{N} & = \begin{cases}-\left(1.1-\sqrt{(x-1.1 \sqrt{0.91})^{2}+y^{2}}\right) & \text { if } x \geq 0 \\
-(0.6+0.3 \cos (6 \theta)-r)) & \text { if } x<0\end{cases} \\
\phi^{R} & =-1 \\
G & =\left(r^{2}-.25\right)^{3} .
\end{aligned}
$$


Figure 6 depicts the solution and highlights different parts of the interface where Dirichlet and nonhomogenous Neumann boundary conditions are enforced. Table 3 demonstrates the second-order accuracy of the method in the $L^{\infty}$-norm.

\begin{tabular}{|c|c|c|}
\hline Resolution & $\left\|u-u_{h}\right\|_{\infty}$ & Order \\
\hline $64^{2}$ & $5.09 \times 10^{-3}$ & - \\
\hline $128^{2}$ & $1.18 \times 10^{-3}$ & 2.11 \\
\hline $256^{2}$ & $3.54 \times 10^{-4}$ & 1.74 \\
\hline $512^{2}$ & $9.34 \times 10^{-5}$ & 1.92 \\
\hline $1024^{2}$ & $2.50 \times 10^{-5}$ & 1.90 \\
\hline
\end{tabular}

Table 3: Maximum error and rate of maximum error for different resolution for Ex. 3.1.3: Mixed Dirichlet and non-homogenous Neumann boundary conditions on irregular interfaces in two spatial dimensions.

\subsubsection{Mixed Dirichlet and non-homogenous Robin boundary conditions}

Consider the Poisson equation on the domain $\Omega=[-1,1] \times[-1,1]$. We define the following for $x, y \in \Omega:$

$$
\begin{aligned}
r & =\sqrt{x^{2}+y^{2}} \\
\theta & =\tan ^{-1}\left(\frac{y}{x}\right) \\
\phi^{D} & = \begin{cases}-\min (r-0.1,0.6+0.3 \cos (6 \theta)-r) & \text { if } x \geq 0 \\
-\min \left(r-0.1,1.1-\sqrt{(x+1.1 \sqrt{0.91})^{2}+y^{2}}\right. & \text { if } x<0\end{cases} \\
\phi^{N} & =-1 ; \\
\phi^{R} & = \begin{cases}-\left(1.1-\sqrt{(x-1.1 \sqrt{0.91})^{2}+y^{2}}\right) & \text { if } x \geq 0 \\
-(0.6+0.3 \cos (6 \theta)-r)) & \text { if } x<0\end{cases} \\
G & =\left(r^{2}-.25\right)^{3}
\end{aligned}
$$


Figure 7 depicts the solution and highlights different parts of the interface where Dirichlet and non-homogenous Robin boundary conditions are enforced. Table 4 demonstrates the second-order accuracy of the method in the $L^{\infty}$-norm.

\begin{tabular}{|c|c|c|}
\hline Resolution & $\left\|u-u_{h}\right\|_{\infty}$ & Order \\
\hline $128^{2}$ & $1.24 \times 10^{-3}$ & - \\
\hline $256^{2}$ & $2.92 \times 10^{-4}$ & 2.09 \\
\hline $512^{2}$ & $8.78 \times 10^{-5}$ & 1.73 \\
\hline $1024^{2}$ & $1.98 \times 10^{-5}$ & 2.15 \\
\hline
\end{tabular}

Table 4: Maximum error and rate of maximum error for different resolution for Ex. 3.1.4: Mixed Dirichlet and non-homogenous Robin boundary conditions on irregular interfaces in two spatial dimensions.

\subsubsection{Mixed non-homogenous Neumann and non-homogenous Robin boundary con- ditions}

Consider the Poisson equation on the domain $\Omega=[-1,1] \times[-1,1]$. We define the following for $x, y \in \Omega:$

$$
\begin{aligned}
r & =\sqrt{(x-0.05)^{2}+(y+0.09)^{2}}, \\
\beta & =\frac{\left((y+0.09)^{5}+5 \cdot(x-0.05)^{4} \cdot(y+0.09)-10 \cdot(x-0.05)^{2} \cdot(y+0.09)^{3}\right)}{r^{5}}, \\
\phi^{D} & =-1 \\
\phi^{N} & =\left(r-0.5-\frac{\beta}{3}\right) \\
\phi^{R} & =x-0.1 \\
G & =\left(\left(x^{2}+y^{2}\right)^{2}-.25\right)^{3} .
\end{aligned}
$$

Figure 8 depicts the solution and highlights different parts of the interface where non-homogenous Neumann and non-homogenous Robin boundary conditions are enforced. Table 5 demonstrates the 
second-order accuracy of the method in the $L^{\infty}$-norm.

\begin{tabular}{|c|c|c|}
\hline Resolution & $\left\|u-u_{h}\right\|_{\infty}$ & Order \\
\hline $64^{2}$ & $6.32 \times 10^{-3}$ & - \\
\hline $128^{2}$ & $1.63 \times 10^{-3}$ & 1.95 \\
\hline $256^{2}$ & $4.44 \times 10^{-4}$ & 1.88 \\
\hline $512^{2}$ & $1.09 \times 10^{-4}$ & 2.03 \\
\hline $1024^{2}$ & $3.13 \times 10^{-5}$ & 1.80 \\
\hline $2048^{2}$ & $7.35 \times 10^{-6}$ & 2.09 \\
\hline
\end{tabular}

Table 5: Maximum error and rate of maximum error for different resolution for Ex. 3.1.5: Mixed nonhomogenous Neumann and non-homogenous Robin boundary conditions on irregular interfaces in two spatial dimensions.

\subsubsection{Mixed Dirichlet, Non-Homogenous Neumann and Non-Homogenous Robin Bound- ary Conditions on Smooth Interfaces}

Consider the Poisson equation on the domain $\Omega=[-1,1] \times[-1,1]$. We define $r=\sqrt{x^{2}+y^{2}}$, $\phi^{D}=-x-.3, \phi^{N}=r-.8, \phi^{R}=-\frac{x+y}{\sqrt{2}}+.1$, and the exact solution $G=\left(r^{4}-.25\right)^{3}$ for all $x, y \in \Omega$. Figure 9 depicts the solution and highlights the different parts of the interface where Dirichlet, non-homegeneous Neumann and non-homogeneous Robin boundary conditions are enforced. Table 6 demonstrates the second-order accuracy of the method in the $L^{\infty}$-norm. 


\begin{tabular}{|c|c|c|}
\hline Resolution & $\left\|u-u_{h}\right\|_{\infty}$ & Order \\
\hline $128^{2}$ & $4.14 \times 10^{-6}$ & - \\
\hline $256^{2}$ & $9.34 \times 10^{-7}$ & 2.10 \\
\hline $512^{2}$ & $2.50 \times 10^{-7}$ & 1.95 \\
\hline $1024^{2}$ & $6.03 \times 10^{-8}$ & 2.05 \\
\hline $2048^{2}$ & $1.74 \times 10^{-8}$ & 1.79 \\
\hline
\end{tabular}

Table 6: Maximum error and rate of maximum error for different resolution for Ex. 3.1.6: Mixed Dirichlet, non-homogenous Neumann and non-homogenous Robin boundary conditions on smooth interfaces in two spatial dimensions.

\subsubsection{Mixed Dirichlet, Non-Homogenous Neumann and Non-Homogenous Robin Bound- ary Conditions on Non-Smooth Interfaces}

Consider the Poisson equation on the domain $\Omega=[-1,1] \times[-1,1]$. We define the following for $x, y \in \Omega:$

$$
\begin{aligned}
r & =\sqrt{(x-0.05)^{2}+(y+0.09)^{2}}, \\
\beta & =\frac{\left((y+0.09)^{5}+5 \cdot(x-0.05)^{4} \cdot(y+0.09)-10 \cdot(x-0.05)^{2} \cdot(y+0.09)^{3}\right)}{r^{5}}, \\
\phi^{D} & =\frac{x+y}{\sqrt{2}}-.1 \\
\phi^{N} & =\left(r-0.5-\frac{\beta}{3}\right) \\
\phi^{R} & =x-0.1 \\
G & =\left(\left(x^{2}+y^{2}\right)^{2}-.25\right)^{3} .
\end{aligned}
$$

Figure 10 depicts the solution and highlights the different parts of the interface where Dirichlet, non-homegeneous Neumann and non-homogeneous Robin boundary conditions are enforced. Table 7 demonstrates the second-order accuracy of the method in the $L^{\infty}$-norm. 

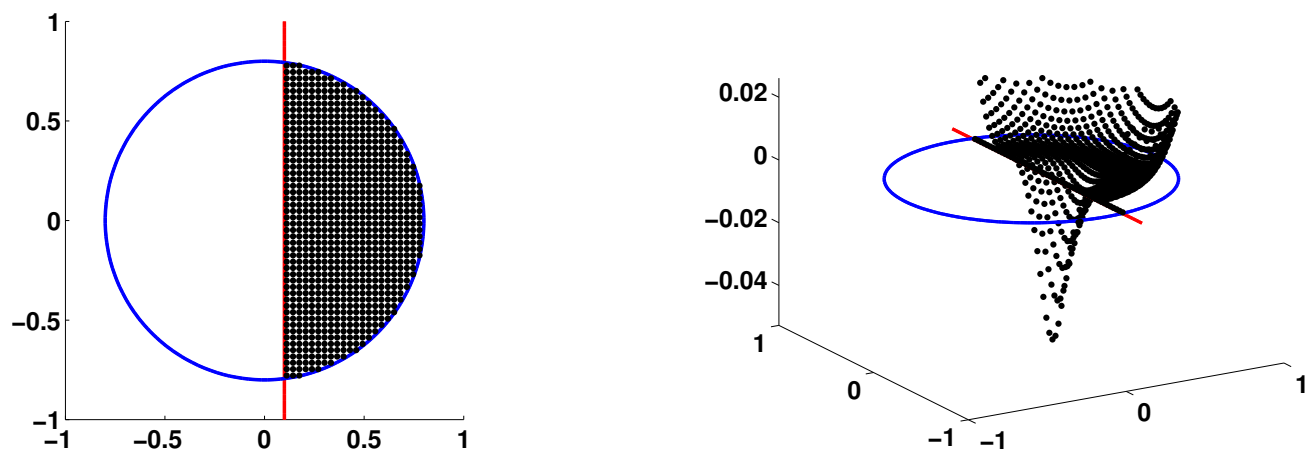

Figure 4: Plot of the solution, $u$, and interfaces for example 3.1.1. The left figure shows a top view where the two interfaces are easily detected. The red line represent $\Gamma^{D}$ and the blue circle represent $\Gamma^{N}$. The right figure shows the solution inside $\Omega^{-}$.
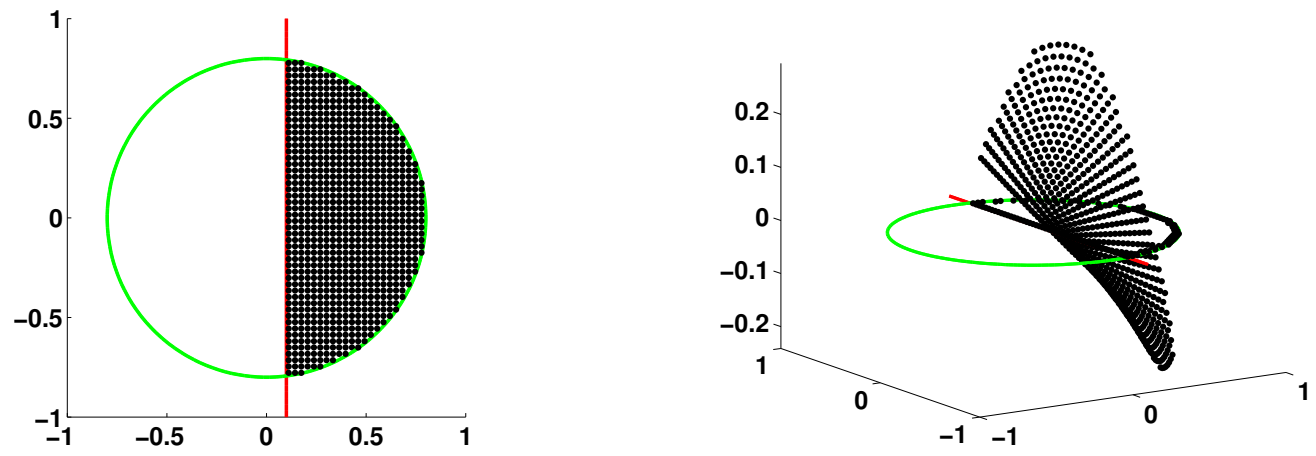

Figure 5: Plot of the solution, $u$, and interfaces for example 3.1.2. The left figure shows a top view where the two interfaces are easily detected. The red line represent $\Gamma^{D}$ and the green circle represent $\Gamma^{R}$. The right figure shows the solution inside $\Omega^{-}$. 

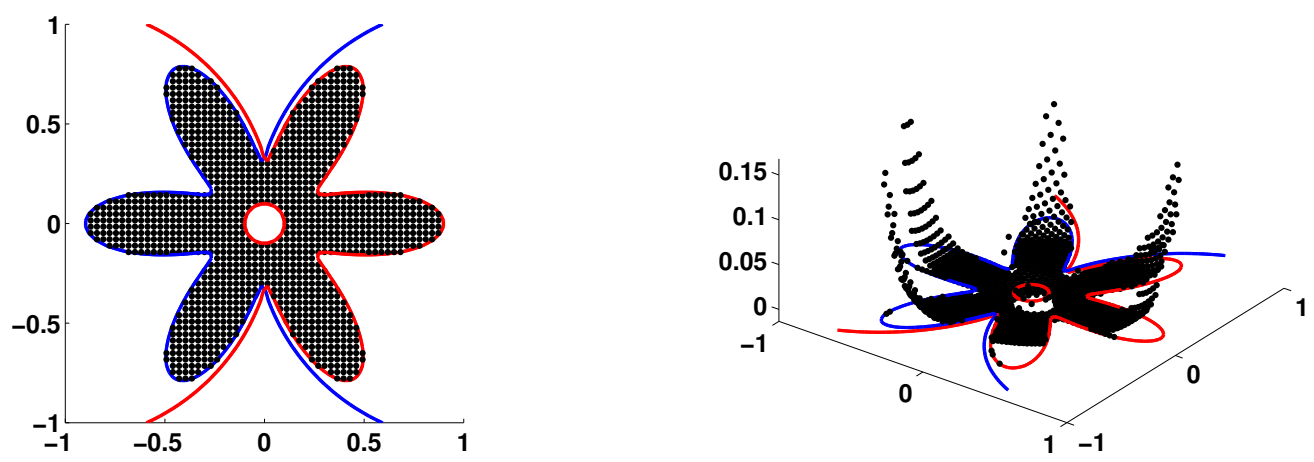

Figure 6: Plot of the solution, $u$, and interfaces for example 3.1.3. The left figure shows a top view where the two interfaces are easily detected. The red curve represents $\Gamma^{D}$ and the blue curve represents $\Gamma^{N}$. The right figure shows the solution inside $\Omega^{-}$.
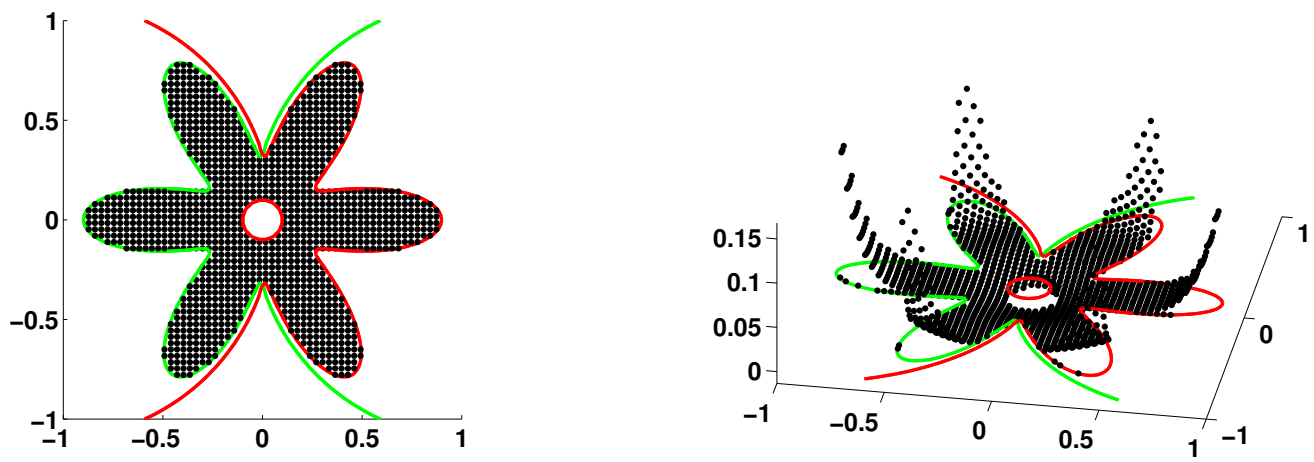

Figure 7: Plot of the solution, $u$, and interfaces for example 3.1.4. The left figure shows a top view where the two interfaces are easily detected. The red curve represents $\Gamma^{D}$ and the green curve represents $\Gamma^{R}$. The right figure shows the solution inside $\Omega^{-}$. 

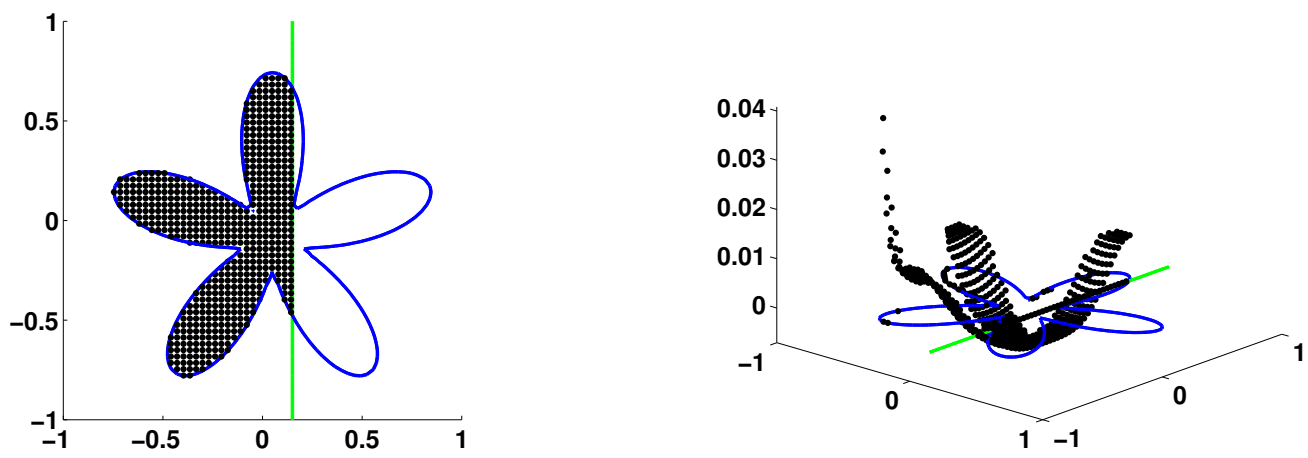

Figure 8: Plot of the solution, $u$, and interfaces for example 3.1.5. The left figure shows a top view where the two interfaces are easily detected. The blue curve represent $\Gamma^{N}$ and the green line represent $\Gamma^{R}$. The right figure shows the solution inside $\Omega^{-}$.
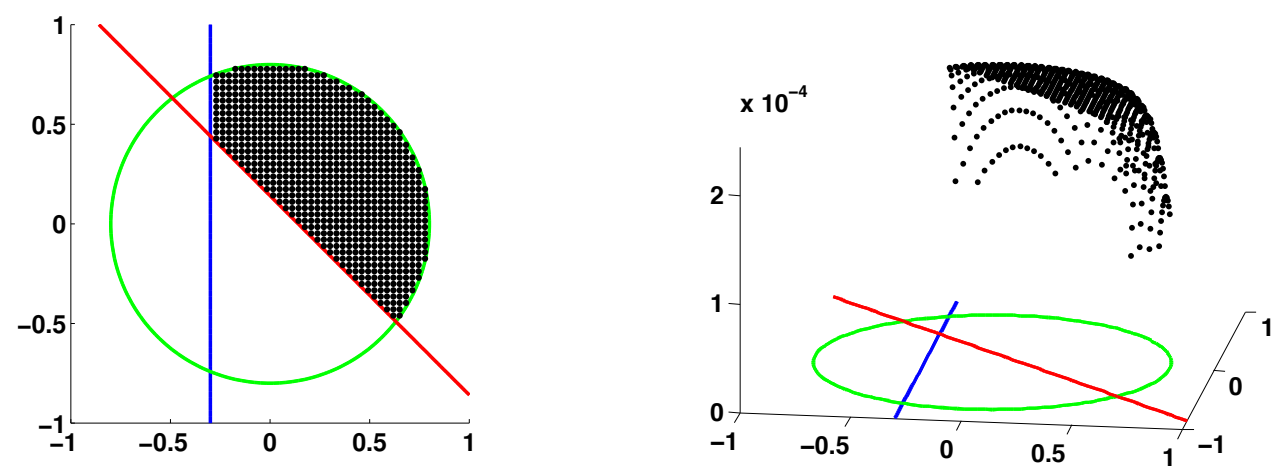

Figure 9: Plot of the solution, $u$, and interfaces for example 3.1.6. The left figure shows a top view where the three interfaces are easily detected. The red line represents $\Gamma^{D}$, the blue line represent $\Gamma^{N}$ and the green circle represent $\Gamma^{R}$. The right figure shows the solution inside $\Omega^{-}$. 


\begin{tabular}{|c|c|c|}
\hline Resolution & $\left\|u-u_{h}\right\|_{\infty}$ & Order \\
\hline $64^{2}$ & $8.10 \times 10^{-3}$ & - \\
\hline $128^{2}$ & $2.13 \times 10^{-3}$ & 1.92 \\
\hline $256^{2}$ & $5.86 \times 10^{-4}$ & 1.86 \\
\hline $512^{2}$ & $1.44 \times 10^{-4}$ & 2.02 \\
\hline $1024^{2}$ & $4.27 \times 10^{-5}$ & 1.75 \\
\hline $2048^{2}$ & $1.04 \times 10^{-5}$ & 2.04 \\
\hline
\end{tabular}

Table 7: Maximum error and rate of maximum error for different resolution for Ex. 3.1.7: Mixed Dirichlet, non-homogenous Neumann and non-homogenous Robin boundary conditions on non-smooth interfaces in two spatial dimensions.
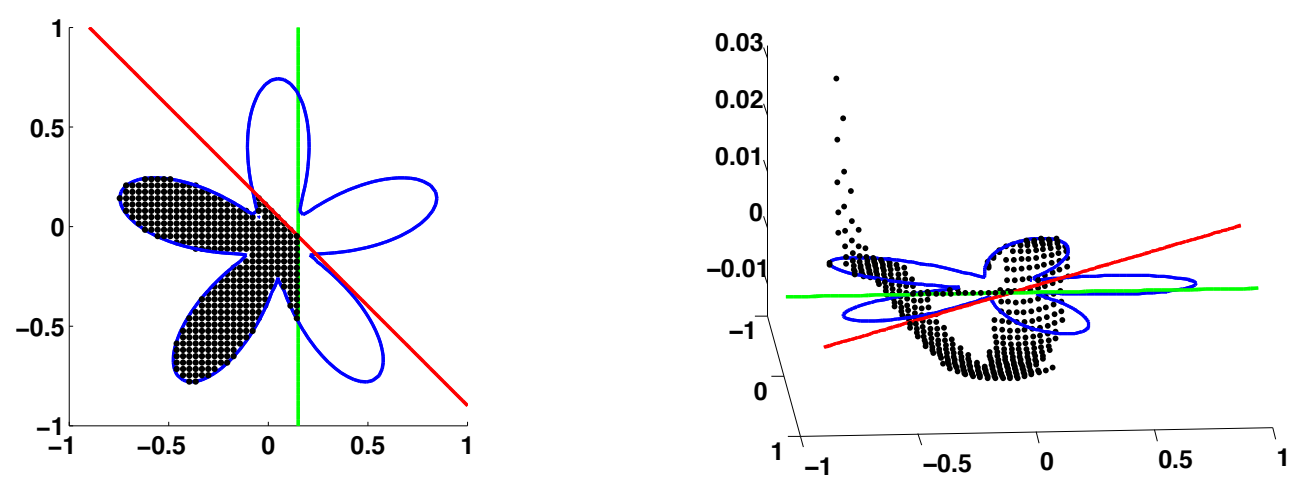

Figure 10: Plot of the solution, $u$, and interfaces for example 3.1.7. The left figure shows a top view where the three interfaces are easily detected. The red line represents $\Gamma^{D}$, the blue curves represent $\Gamma^{N}$ and the green line represent $\Gamma^{R}$. The right figure shows the solution inside $\Omega^{-}$. 


\subsection{Three Spatial Dimensions}

\subsubsection{Mixed Dirichlet and non-homogenous Neumann boundary conditions}

Consider the Poisson equation on the domain $\Omega=[-1,1] \times[-1,1] \times[-1,1]$. We define $r=$ $\sqrt{x^{2}+y^{2}+z^{2}}, \phi^{D}=-x+.1, \phi^{N}=r-.8, \phi^{R}=-1$, and the exact solution $G=\left(r^{2}-.25\right)^{3}$ for all $x, y, z \in \Omega$. Figure 11 depicts the different parts of the interface where Dirichlet and nonhomogeneous Neumann boundary conditions are enforced. Table 8 shows the second-order accuracy of the method in the $L^{\infty}$.

\begin{tabular}{|c|c|c|}
\hline Resolution & $\left\|u-u_{h}\right\|_{\infty}$ & Order \\
\hline $16^{3}$ & $2.55 \times 10^{-2}$ & - \\
\hline $32^{3}$ & $5.15 \times 10^{-3}$ & 2.31 \\
\hline $64^{3}$ & $1.43 \times 10^{-3}$ & 1.85 \\
\hline $128^{3}$ & $4.54 \times 10^{-4}$ & 1.66 \\
\hline
\end{tabular}

Table 8: Maximum error and rate of maximum error for different resolution for Ex. 3.2.1: Mixed Dirichlet and non-homogenous Neumann boundary conditions on smooth interfaces in three spatial dimensions.

\subsubsection{Mixed Dirichlet and non-homogenous Robin boundary conditions}

Consider the Poisson equation on the domain $\Omega=[-1,1] \times[-1,1] \times[-1,1]$. We define $r=$ $\sqrt{x^{2}+y^{2}+z^{2}}, \phi^{D}=-x+.1, \phi^{N}=-1, \phi^{R}=r-.5$, and the exact solution $G=\left(r^{2}-.25\right)^{3}$ for all $x, y, z \in \Omega$. Figure 12 depicts the different parts of the interface where Dirichlet and nonhomogeneous Robin boundary conditions are enforced. Table 9 shows the second-order accuracy of the method in the $L^{\infty}$.

\subsubsection{Mixed non-homogenous Neumann and non-homogenous Robin boundary con- ditions}

Consider the Poisson equation on the domain $\Omega=[-1,1] \times[-1,1] \times[-1,1]$. We define $r=$ $\sqrt{x^{2}+y^{2}+z^{2}}, \phi^{D}=-1 ., \phi^{N}=-x+.1, \phi^{R}=r-.8$, and the exact solution $G=\left(r^{2}-.25\right)^{3}$ for all 


\begin{tabular}{|c|c|c|}
\hline Resolution & $\left\|u-u_{h}\right\|_{\infty}$ & Order \\
\hline $16^{3}$ & $2.52 \times 10^{-4}$ & - \\
\hline $32^{3}$ & $6.83 \times 10^{-5}$ & 1.88 \\
\hline $64^{3}$ & $1.78 \times 10^{-5}$ & 1.84 \\
\hline $128^{3}$ & $4.53 \times 10^{-6}$ & 1.98 \\
\hline
\end{tabular}

Table 9: Maximum error and rate of maximum error for different resolution for Ex. 3.2.2: Mixed Dirichlet and non-homogenous Robin boundary conditions on smooth interfaces in three spatial dimensions.

$x, y, z \in \Omega$. Figure 13 depicts the different parts of the interface where non-homegeneous Neumann and non-homogeneous Robin boundary conditions are enforced. Table 10 shows the second-order accuracy of the method in the $L^{\infty}$.

\begin{tabular}{|c|c|c|}
\hline Resolution & $\left\|u-u_{h}\right\|_{\infty}$ & Order \\
\hline $16^{3}$ & $3.12 \times 10^{-2}$ & - \\
\hline $32^{3}$ & $1.04 \times 10^{-2}$ & 1.58 \\
\hline $64^{3}$ & $2.97 \times 10^{-3}$ & 1.81 \\
\hline $128^{3}$ & $8.39 \times 10^{-4}$ & 1.83 \\
\hline
\end{tabular}

Table 10: Maximum error and rate of maximum error for different resolution for Ex. 3.2.3: Mixed non-homogenous Neumann and non-homogenous Robin boundary conditions on smooth interfaces in three spatial dimensions.

\subsubsection{Mixed non-homogenous Neumann and non-homogenous Robin boundary con- ditions}

Consider the Poisson equation on the domain $\Omega=[-1,1] \times[-1,1] \times[-1,1]$. We define $r=$ $\sqrt{x^{2}+y^{2}+z^{2}}, \phi^{D}=-1 ., \phi^{N}=r-.8, \phi^{R}=-x+.1$, and the exact solution $G=\left(r^{2}-.25\right)^{3}$ for all $x, y, z \in \Omega$. Figure 14 depicts the different parts of the interface where non-homegeneous Neumann and non-homogeneous Robin boundary conditions are enforced. Table 11 shows the second-order 
accuracy of the method in the $L^{\infty}$.

\begin{tabular}{|c|c|c|}
\hline Resolution & $\left\|u-u_{h}\right\|_{\infty}$ & Order \\
\hline $16^{3}$ & $2.71 \times 10^{-2}$ & - \\
\hline $32^{3}$ & $9.55 \times 10^{-3}$ & 1.51 \\
\hline $64^{3}$ & $2.75 \times 10^{-3}$ & 1.80 \\
\hline $128^{3}$ & $7.89 \times 10^{-4}$ & 1.80 \\
\hline
\end{tabular}

Table 11: Maximum error and rate of maximum error for different resolution for Ex. 3.2.4: Mixed non-homogenous Neumann and non-homogenous Robin boundary conditions on smooth interfaces in three spatial dimensions.

\subsubsection{Mixed non-homogenous Neumann and non-homogenous Robin boundary con- ditions}

Consider the Poisson equation on the domain $\Omega=[-1,1] \times[-1,1] \times[-1,1]$. We define $r=$ $\sqrt{x^{2}+y^{2}+z^{2}}, \phi^{D}=-1 ., \phi^{N}=-x+.1, \phi^{R}=r-.5$, and the exact solution $G=\left(r^{2}-.25\right)^{3}$ for all $x, y, z \in \Omega$. Figure 15 depicts the different parts of the interface where non-homegeneous Neumann and non-homogeneous Robin boundary conditions are enforced. Table 12 shows the second-order accuracy of the method in the $L^{\infty}$.

\begin{tabular}{|c|c|c|}
\hline Resolution & $\left\|u-u_{h}\right\|_{\infty}$ & Order \\
\hline $16^{3}$ & $8.62 \times 10^{-4}$ & - \\
\hline $32^{3}$ & $2.10 \times 10^{-4}$ & 2.04 \\
\hline $64^{3}$ & $6.04 \times 10^{-5}$ & 1.80 \\
\hline $128^{3}$ & $1.43 \times 10^{-5}$ & 2.08 \\
\hline
\end{tabular}

Table 12: Maximum error and rate of maximum error for different resolution for Ex. 3.2.5: Mixed non-homogenous Neumann and non-homogenous Robin boundary conditions on smooth interfaces in three spatial dimensions. 


\subsubsection{Mixed Dirichlet, Non-Homogenous Neumann And Non-Homogenous Robin Boundary Conditions on Smooth Interfaces}

Consider the Poisson equation on the domain $\Omega=[-1,1] \times[-1,1] \times[-1,1]$. We define $r=$ $\sqrt{x^{2}+y^{2}+z^{2}}, \phi^{D}=-\frac{x+y}{\sqrt{2}}+.1, \phi^{N}=r-.8, \phi^{R}=-x+.1$, and the exact solution $G=\left(r^{2}-.25\right)^{4}$ for all $x, y \in \Omega$. Figure 16 depicts the different parts of the interface where Dirichlet, non-homegeneous Neumann and non-homogeneous Robin boundary conditions are enforced. Table 13 shows the second-order accuracy of the method in the $L^{\infty}$.

\begin{tabular}{|c|c|c|}
\hline Resolution & $\left\|u-u_{h}\right\|_{\infty}$ & Order \\
\hline $16^{3}$ & $5.74 \times 10^{-2}$ & - \\
\hline $32^{3}$ & $1.66 \times 10^{-2}$ & 1.79 \\
\hline $64^{3}$ & $4.42 \times 10^{-3}$ & 1.91 \\
\hline $128^{3}$ & $1.06 \times 10^{-3}$ & 2.06 \\
\hline
\end{tabular}

Table 13: Maximum error and rate of maximum error for different resolution for Ex. 3.2.6: Mixed Dirichlet, non-homogenous Neumann and non-homogenous Robin boundary conditions on smooth interfaces in three spatial dimensions. 


\subsubsection{Mixed Dirichlet, Non-Homogenous Neumann And Non-Homogenous Robin Boundary Conditions on Non-Smooth Interfaces}

Consider a domain $\Omega=[-1,1] \times[-1,1] \times[-1,1]$. We define the following for $x, y, z \in \Omega$ :

$$
\begin{aligned}
\phi^{D} & =-\frac{x+y+z}{\sqrt{3}}-0.1, \\
\phi^{N} & =x-0.4, \\
a & =\sqrt{0.4}-\sqrt{(x+0.05)^{2}+(y+0.1)^{2}+(z+0.05)^{2}}, \\
b & =\sqrt{0.12}-\sqrt{(x+0.05)^{2}+\frac{(y+0.1)^{2}}{5}+(z+0.1)^{2}}, \\
c & =\sqrt{0.12}-\sqrt{\frac{(x+0.05)^{2}}{5}+(y+0.1)^{2}+(z+0.1)^{2}}, \\
d & =\sqrt{\sqrt{2} \cdot 0.24}-\sqrt{2 x^{2}+2 y^{2}+\frac{z^{2}}{2}}, \\
\phi^{R} & =-\max (a, \max (b, \max (c, d))), \\
G & =\left(r^{2}-.25\right)^{4} .
\end{aligned}
$$

Figure 17 depicts the interfaces where the boundary conditions are imposed. Table 14 shows the second-order accuracy of the method in the $L^{\infty}$-norm.

\begin{tabular}{|c|c|c|}
\hline Resolution & $\left\|u-u_{h}\right\|_{\infty}$ & Order \\
\hline $16^{3}$ & $1.10 \times 10^{-2}$ & - \\
\hline $32^{3}$ & $2.75 \times 10^{-3}$ & 2.00 \\
\hline $64^{3}$ & $5.71 \times 10^{-4}$ & 2.27 \\
\hline $128^{3}$ & $1.87 \times 10^{-4}$ & 1.61 \\
\hline
\end{tabular}

Table 14: Maximum error and rate of maximum error for different resolution for Ex. 3.2.7: Mixed Dirichlet, non-homogenous Neumann and non-homogenous Robin boundary conditions on non-smooth interfaces in three spatial dimensions. 


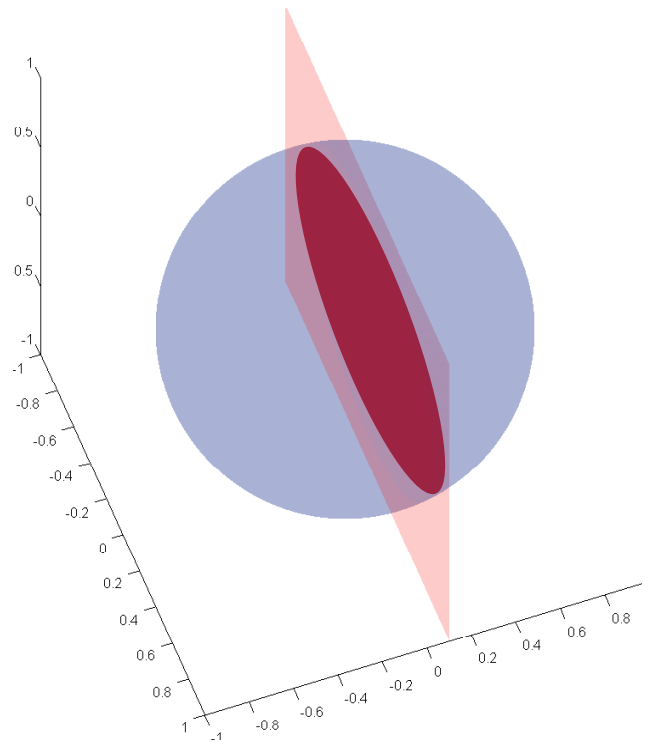

Figure 11: Plot of the two interfaces for example 3.2.1. The red plane represents $\Gamma^{D}$ and the blue sphere represents $\Gamma^{N}$. The top part of the blue sphere and the darkened red circle mark the boundary of $\Omega^{-}$.

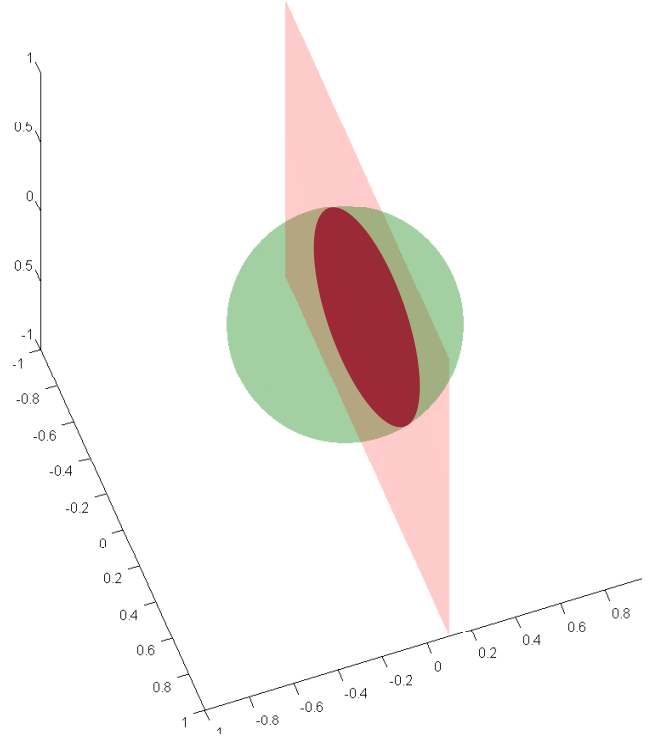

Figure 12: Plot of the two interfaces for example 3.2.2. The red plane represents $\Gamma^{D}$ and the green sphere represents $\Gamma^{R}$. The top part of the green sphere and the darkened red circle mark the boundary of $\Omega^{-}$. 


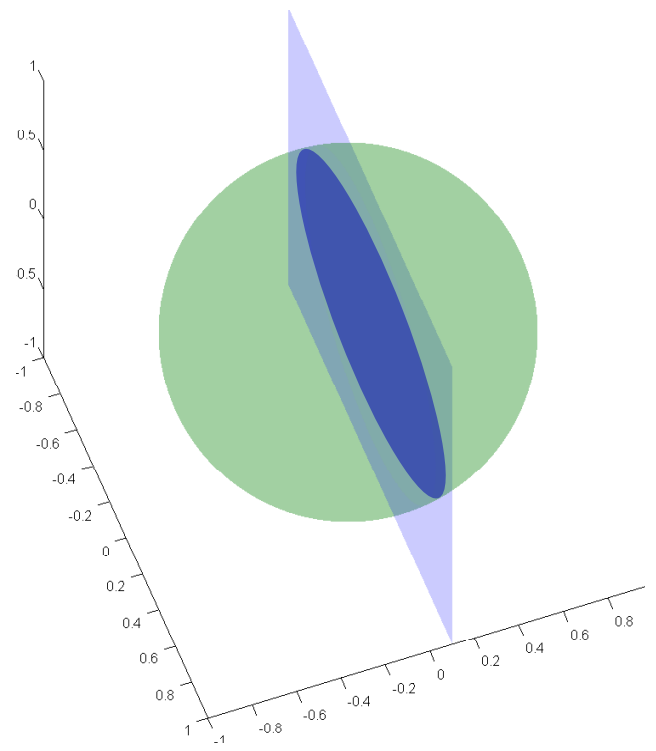

Figure 13: Plot of the two interfaces for example 3.2.3. The blue plane represents $\Gamma^{N}$ and the green sphere represents $\Gamma^{R}$. The top part of the green sphere, and the darkened blue circle mark the boundary of $\Omega^{-}$. 


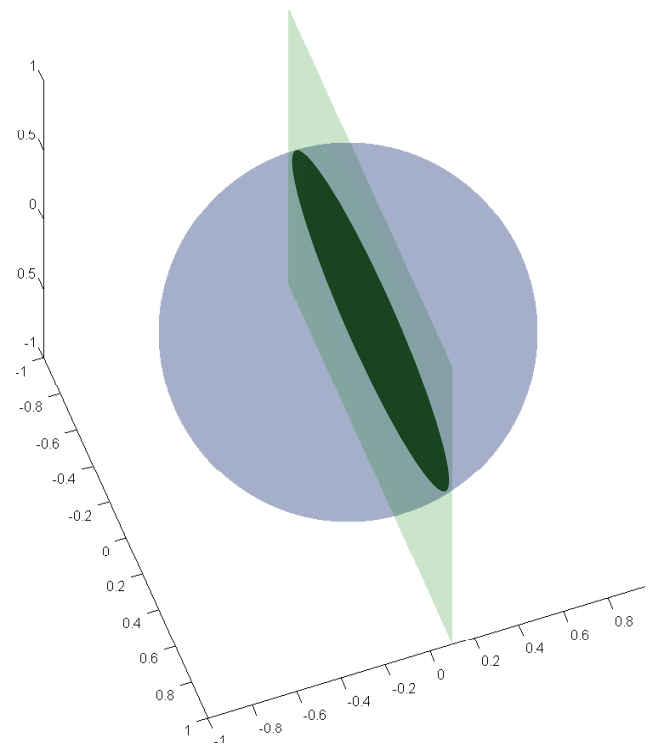

Figure 14: Plot of the two interfaces for example 3.2.4. The blue sphere represents $\Gamma^{N}$ and the green plane represents $\Gamma^{R}$. The top part of the blue sphere and the darkened green circle mark the boundary of $\Omega^{-}$. 


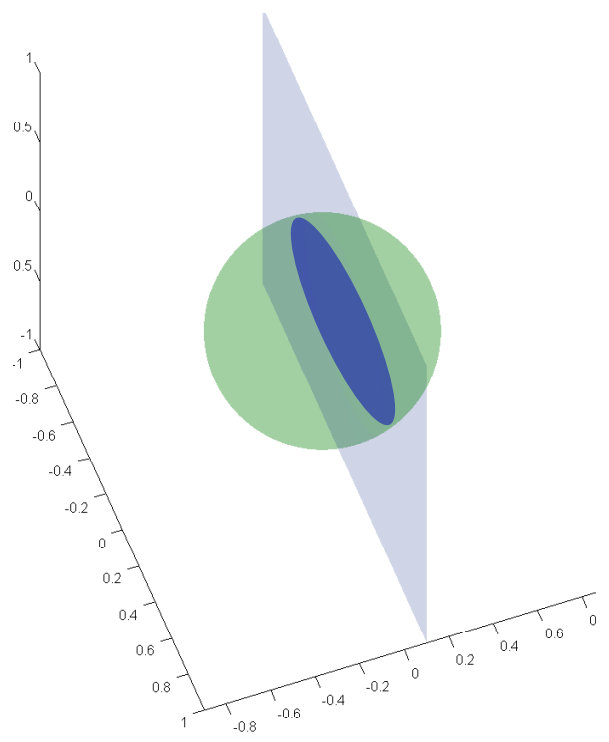

Figure 15: Plot of the two interfaces for example 3.2.5. The blue plane represents $\Gamma^{N}$ and the green sphere represents $\Gamma^{R}$. The top part of the green sphere and the darkened blue circle mark the boundary of $\Omega^{-}$.

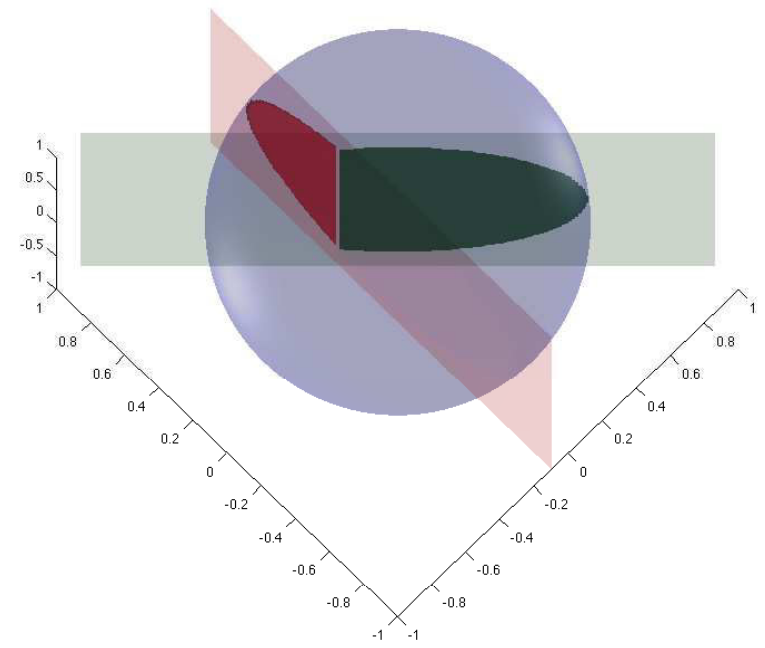

Figure 16: Plot of the three interfaces for example 3.2.6. The red plane represents $\Gamma^{D}$, the blue sphere represents $\Gamma^{N}$ and the green plane represents $\Gamma^{R}$. The top part of the blue sphere, the darkened red and the darkened green interfaces mark the boundary of $\Omega^{-}$. 


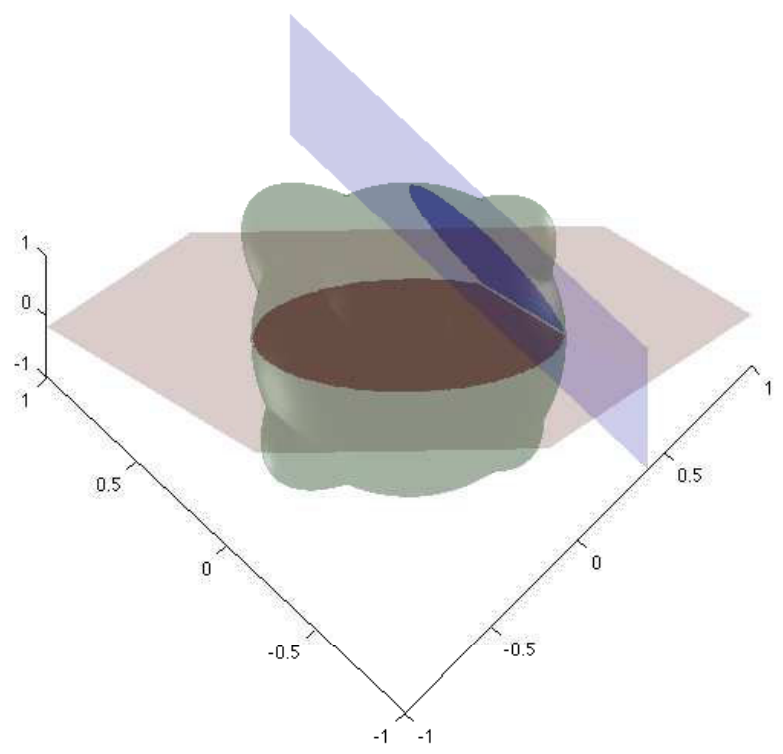

Figure 17: Plot of the three interfaces for example 3.2.7. The red plane represents $\Gamma^{D}$, the blue plane represents $\Gamma^{N}$ and the green curved surface represents $\Gamma^{R}$. The top left part of the green curved plane, the darkened red and the darkened blue interfaces mark the boundary of $\Omega^{-}$. 


\section{Remark on robustness of the method}

For clarification purposes we want to point out that two of the boundary conditions always meet at some point, in two dimensions, or at some surface, in three dimensions, in the domain and the method is designed to handle the boundary conditions correctly at those points/surfaces. If all three boundary conditions are present in the same cell that does not pose a problem, since the level set function with the highest value is always used to determine what boundary conditions are enforced in that direction. If two level set functions are very close to each other over a long stretch that does not pose a problem either since the level set function with the higher value is always chosen as the boundary. In order to emphasize this we show a few two dimensional examples where two boundary conditions are close to one another for a long stretch (see Examples 4.1, 4.2 and 4.3) and a two dimensional example where all three boundary conditions are very close (see Example 4.4). If there are two level set functions with the same level set value (or the difference in their value is less than machine precision) at a certain point we would potentially run into problems (it is not clear which boundary condition would be chosen at this specific point), but physically that would equal enforcing two types of boundary conditions at the same boundary which is unphysical so the problem would be illposed. Here below are examples showing the robustness of the method in tough situations. Convergence rate of the conjugate gradient solver is the similar to the convergence rate for the problems tested previously in this paper.

\subsection{Mixed Dirichlet, Non-Homogenous Neumann Level set functions' zeroth level close for long stretches}

Consider the Poisson equation on the domain $\Omega=[-1,1] \times[-1,1]$. We define $r=\sqrt{x^{2}+y^{2}}$, $\phi^{D}=\sqrt{(x-0.0035)^{2}+(y-0.0045)^{2}}-0.8, \phi^{N}=r-.8, \phi^{R}=-1 .$, and the exact solution

$G=\left(\frac{x^{3}}{3}-\frac{x^{2}}{2}\right) \cdot\left(\frac{y^{3}}{3}-\frac{y^{2}}{2}\right)$. The two points that have the zeroth level set furthest away from each other are about 0.0057 away from each other (which is considerably lower than the cell size until the $512^{2}$ resolution is reached), all other points have an even shorter distance between the two interfaces. Figure 18 depicts the solution and highlights the different parts of the interface where Dirichlet and non-homegeneous Neumann boundary conditions are enforced. Table 15 demonstrates 
the second-order accuracy of the method in the $L^{\infty}$-norm. The method maintains second order accuracy despite the zeroth level of the two level set functions being very close over long stretches.

\begin{tabular}{|c|c|c|c|}
\hline Resolution & $\mathrm{dx}$ & $\left\|u-u_{h}\right\|_{\infty}$ & Order \\
\hline $16^{2}$ & 0.125 & $1.72 \times 10^{-3}$ & - \\
\hline $32^{2}$ & 0.0625 & $3.10 \times 10^{-4}$ & 2.47 \\
\hline $64^{2}$ & 0.03125 & $1.13 \times 10^{-4}$ & 1.49 \\
\hline $128^{2}$ & 0.015625 & $2.15 \times 10^{-5}$ & 2.39 \\
\hline $256^{2}$ & 0.0078125 & $5.47 \times 10^{-6}$ & 1.98 \\
\hline $512^{2}$ & 0.00390625 & $1.32 \times 10^{-6}$ & 2.05 \\
\hline $1024^{2}$ & 0.001953125 & $3.29 \times 10^{-7}$ & 2.00 \\
\hline
\end{tabular}

Table 15: Maximum error and rate of maximum error for different resolution for Ex. 4.1: Mixed Dirichlet and non-homogenous Neumann boundary conditions on interfaces in two spatial dimensions that are very close to each other over long stretches.

\subsection{Mixed Dirichlet, Non-Homogenous Robin Level set functions' zeroth level close for long stretches}

Consider the Poisson equation on the domain $\Omega=[-1,1] \times[-1,1]$. We define $r=\sqrt{x^{2}+y^{2}}$, $\phi^{D}=\sqrt{(x-0.0035)^{2}+(y-0.0045)^{2}}-0.8, \phi^{N}=-1 ., \phi^{R}=r-.8$, and the exact solution $G=\left(\frac{x^{3}}{3}-\frac{x^{2}}{2}\right) \cdot\left(\frac{y^{3}}{3}-\frac{y^{2}}{2}\right)$. The two points that have the zeroth level set furthest away from each other are about 0.0057 away from each other (which is considerably lower than the cell size until the $512^{2}$ resolution is reached), all other points have an even shorter distance between the two interfaces. Figure 19 depicts the solution and highlights the different parts of the interface where Dirichlet and non-homegeneous Robin boundary conditions are enforced. Table 16 demonstrates the second-order accuracy of the method in the $L^{\infty}$-norm. The method maintains second order accuracy despite the zeroth level of the two level set functions being very close over long stretches. 


\begin{tabular}{|c|c|c|c|}
\hline Resolution & $\mathrm{dx}$ & $\left\|u-u_{h}\right\|_{\infty}$ & Order \\
\hline $16^{2}$ & 0.125 & $1.93 \times 10^{-3}$ & - \\
\hline $32^{2}$ & 0.0625 & $3.51 \times 10^{-4}$ & 2.46 \\
\hline $64^{2}$ & 0.03125 & $1.18 \times 10^{-4}$ & 1.58 \\
\hline $128^{2}$ & 0.015625 & $2.23 \times 10^{-5}$ & 2.40 \\
\hline $256^{2}$ & 0.0078125 & $5.21 \times 10^{-6}$ & 2.10 \\
\hline $512^{2}$ & 0.00390625 & $1.26 \times 10^{-6}$ & 2.05 \\
\hline $1024^{2}$ & 0.001953125 & $3.27 \times 10^{-7}$ & 1.95 \\
\hline
\end{tabular}

Table 16: Maximum error and rate of maximum error for different resolution for Ex. 4.2: Mixed Dirichlet and non-homogenous Robin boundary conditions on interfaces in two spatial dimensions that are very close to each other over long stretches.

\subsection{Mixed Non-Homogenous Neumann and Non-Homogenous Robin Level set functions' zeroth level close for long stretches}

Consider the Poisson equation on the domain $\Omega=[-1,1] \times[-1,1]$. We define $r=\sqrt{x^{2}+y^{2}}$, $\phi^{D}=-1 ., \phi^{N}=\sqrt{(x-0.0035)^{2}+(y-0.0045)^{2}}-0.8, \phi^{R}=r-.8$, and the exact solution $G=\left(\frac{x^{3}}{3}-\frac{x^{2}}{2}\right) \cdot\left(\frac{y^{3}}{3}-\frac{y^{2}}{2}\right)$. The two points that have the zeroth level set furthest away from each other are about 0.0057 away from each other (which is considerably lower than the cell size until the $512^{2}$ resolution is reached), all other points have an even shorter distance between the two interfaces. Figure 20 depicts the solution and highlights the different parts of the interface where non-homegeneous Neumann and non-homegeneous Robin boundary conditions are enforced. Table 17 demonstrates the second-order accuracy of the method in the $L^{\infty}$-norm. The method maintains second order accuracy despite the zeroth level of the two level set functions being very close over long stretches. 


\begin{tabular}{|c|c|c|c|}
\hline Resolution & $\mathrm{dx}$ & $\left\|u-u_{h}\right\|_{\infty}$ & Order \\
\hline $32^{2}$ & 0.0625 & $5.57 \times 10^{-4}$ & - \\
\hline $64^{2}$ & 0.03125 & $1.54 \times 10^{-4}$ & 1.85 \\
\hline $128^{2}$ & 0.015625 & $5.96 \times 10^{-5}$ & 1.37 \\
\hline $256^{2}$ & 0.0078125 & $1.11 \times 10^{-5}$ & 2.42 \\
\hline $512^{2}$ & 0.00390625 & $3.02 \times 10^{-6}$ & 1.88 \\
\hline $1024^{2}$ & 0.001953125 & $7.76 \times 10^{-7}$ & 1.96 \\
\hline
\end{tabular}

Table 17: Maximum error and rate of maximum error for different resolution for Ex. 4.3: Mixed nonhomogenous Neumann and non-homogenous Robin boundary conditions on interfaces in two spatial dimensions that are very close to each other over long stretches.

\subsection{Mixed Dirichlet Non-Homogenous Neumann and Non-Homogenous Robin where all three boundaries are very close}

Consider the Poisson equation on the domain $\Omega=[-1,1] \times[-1,1]$. We define $r=\sqrt{x^{2}+y^{2}}, \phi^{D}=$ $\sqrt{x^{2}+(y-0.1)^{2}}-.395, \phi^{N}=\sqrt{(x-0.3)^{2}+(y-0.1)^{2}}-0.5, \phi^{R}=\sqrt{(x+0.3)^{2}+(y-0.1)^{2}}-0.5$, and the exact solution $G=\left(\frac{x^{3}}{3}-\frac{x^{2}}{2}\right) \cdot\left(\frac{y^{3}}{3}-\frac{y^{2}}{2}\right)$. All three boundary conditions cross within a very small region. The distance between the Dirichlet/Neumann crossings and Dirichlet/Robin crossings is 0.006625 which is significantly lower than the cell resolution until $512^{2}$ is reached. Where as the distance between the Neumann/Robin crossings and each of the other crossings is approx. 0.008334 which is significantly lower than the cell resolution until $256^{2}$ is reached. Figure 21 depicts the solution and highlights the different parts of the interface where Dirichlet, nonhomegeneous Neumann and non-homegeneous Robin boundary conditions are enforced. Table 18 demonstrates the second-order accuracy of the method in the $L^{\infty}$-norm. The method maintains second order accuracy despite the fact that all three level set functions cross very close to each other at two regions in the domain. 

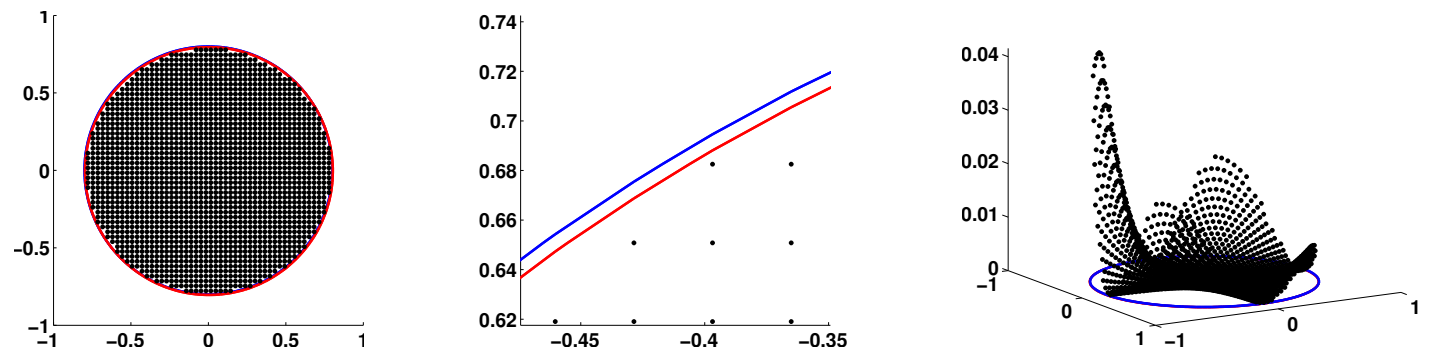

Figure 18: Plot of the solution, $u$, and interfaces for example 4.1. The left figure shows a top view where the two interfaces are easily detected. The red circle represents $\Gamma^{D}$ and the blue circle represents $\Gamma^{N}$. The figure in the middle shows a zoom in to one of the two points that are furthest away from each other. The right figure shows the solution inside $\Omega^{-}$.
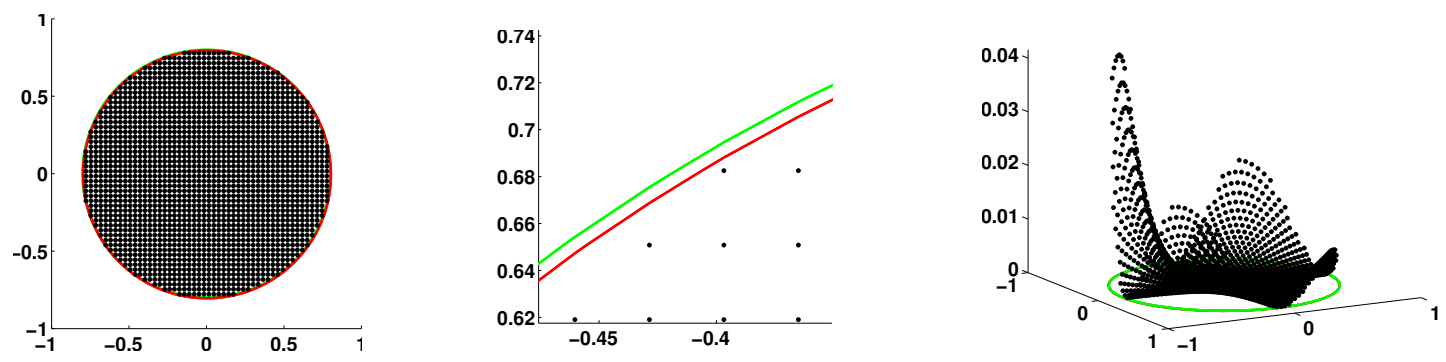

Figure 19: Plot of the solution, $u$, and interfaces for example 4.2. The left figure shows a top view where the two interfaces are easily detected. The red circle represents $\Gamma^{D}$ and the green circle represents $\Gamma^{R}$. The figure in the middle shows a zoom in to one of the two points that are furthest away from each other. The right figure shows the solution inside $\Omega^{-}$. 

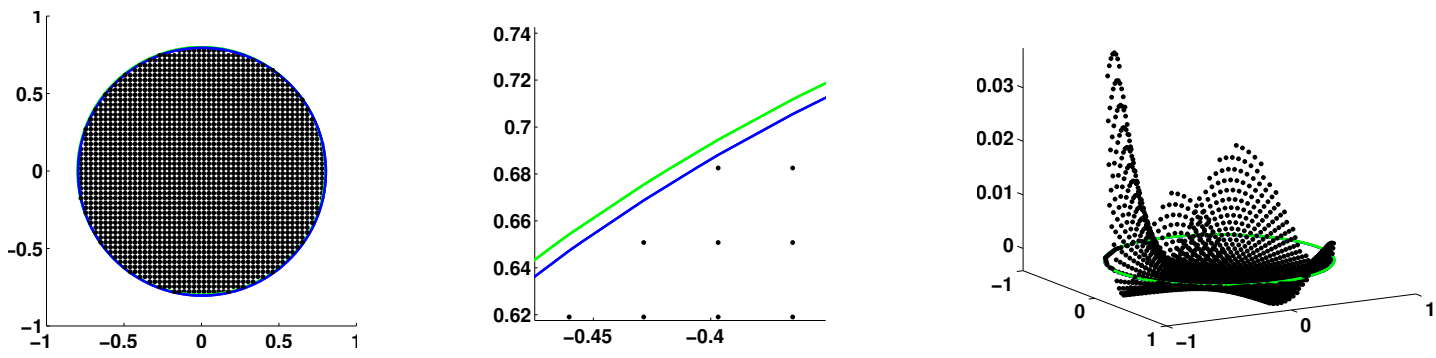

Figure 20: Plot of the solution, $u$, and interfaces for example 4.3. The left figure shows a top view where the two interfaces are easily detected. The blue circle represents $\Gamma^{N}$ and the green circle represents $\Gamma^{R}$. The figure in the middle shows a zoom in to one of the two points that are furthest away from each other. The right figure shows the solution inside $\Omega^{-}$.
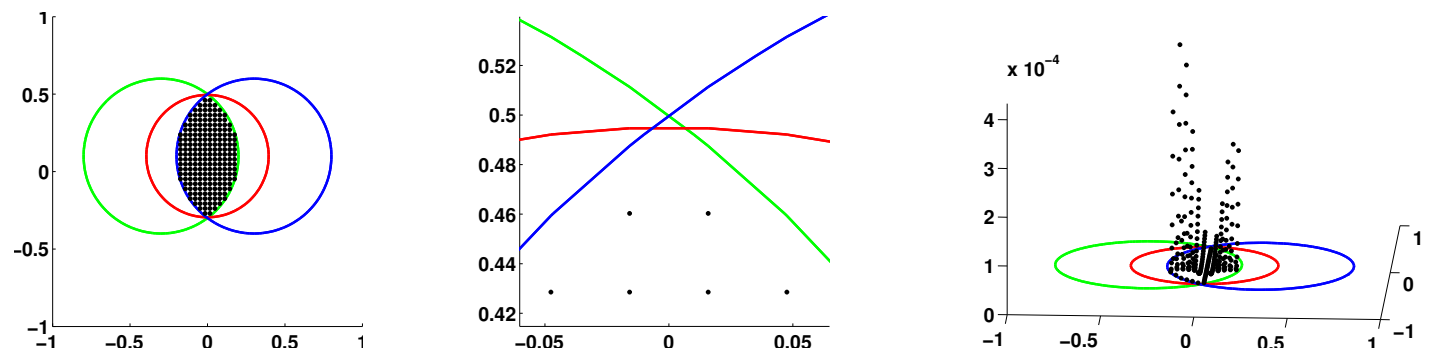

Figure 21: Plot of the solution, $u$, and interfaces for example 4.4. The left figure shows a top view where the three interfaces are easily detected. The red circle represents $\Gamma^{D}$, the blue circle represents $\Gamma^{N}$ and the green circle represents $\Gamma^{R}$. The figure in the middle shows a zoom in to one of the the two points where all level set functions are closest. The right figure shows the solution inside $\Omega^{-}$. 


\begin{tabular}{|c|c|c|c|}
\hline Resolution & $\mathrm{dx}$ & $\left\|u-u_{h}\right\|_{\infty}$ & Order \\
\hline $32^{2}$ & 0.0625 & $3.25 \times 10^{-5}$ & - \\
\hline $64^{2}$ & 0.03125 & $6.85 \times 10^{-6}$ & 2.25 \\
\hline $128^{2}$ & 0.015625 & $2.08 \times 10^{-6}$ & 1.72 \\
\hline $256^{2}$ & 0.0078125 & $7.90 \times 10^{-7}$ & 1.40 \\
\hline $512^{2}$ & 0.00390625 & $1.17 \times 10^{-7}$ & 2.75 \\
\hline $1024^{2}$ & 0.001953125 & $3.01 \times 10^{-8}$ & 1.96 \\
\hline $2048^{2}$ & 0.0009765625 & $1.02 \times 10^{-8}$ & 1.56 \\
\hline
\end{tabular}

Table 18: Maximum error and rate of maximum error for different resolution for Ex. 4.4: Mixed Dirichlet, non-homogenous Neumann and non-homogenous Robin boundary conditions on interfaces in two spatial dimensions where all three boundaries are close.

\section{Conclusion}

We have presented a simple and efficient discretization of the Poisson equation on irregular domains with mixed Dirichlet, Neumann and Robin boundary conditions. This method is straightforward to implement, produces second-order accurate solutions in the $L^{\infty}$-norm and a symmetric positive definite linear system in both two and three spatial dimensions. The method is found to be robust in challenging configurations.

\section{Acknowledgements}

The research of Á. Helgadóttir, Y.T. Ng and F. Gibou were supported in part by ONR under grant agreement N00014-11-1-0027, by the National Science Foundation under grant agreement CHE 1027817 and by the W.M. Keck Foundation. The research of C. Min was supported in part by the Kyung Hee University Research Fund (KHU-20070608) in 2007 and by the Korea Research Foundation Grant funded by the Korean Government (MOEHRD, Basic Research Promotion Fund) (KRF-2008-331-C00045). 


\section{References}

[1] I. Babuska. The finite element method for elliptic with discontinuous coefficients. Commputing, 5:207-213, 1970.

[2] J. Beale and A. Layton. On the accuracy of finite difference methods for elliptic problems with interfaces. Commun. Appl. Math. Comput., 1:207-208, 2006.

[3] J. Bedrossian, J.H. von Brecht, S. Zhu, E. Sifakis, and J.M. Teran. A second order virtual node method for elliptic problems with interfaces and irregular domains. J. Comput. Phys., 229:6405-6426, 2010.

[4] J. Bramble and J. King. A finite element method for interface problems in domains with smooth boundaries and interfaces. Adv. Comput. Math., 6:109-138, 1996.

[5] Z. Chen and J. Zou. Finite element methods, based on nitsche's, method for elliptic interface problems. Numer. Math., 79:175 - 202, 1998.

[6] I.-L. Chern and Y.-C. Shu. A coupling interface method for elliptic interface problems. $J$. Comput. Phys., 225:2138 - 2174, 2007.

[7] A. Coco and G. Russo. A Finite Difference Ghost-cell Multigrid approach for Poisson Equation with mixed Boundary Conditions in Arbitrary Domain. J. Comput. Phys., in press.

[8] J. Dolbow and I. Harari. An efficient finite element method for embedded interfaces problems. J. Numer. Methods Eng., 78:229 - 252, 2009.

[9] M. Dryja. A neumann-neumann algorithm for a mortar discretization of elliptic problems with discontinuous coefficients. J. Numer. Methods Eng., 99:645 - 656, 2005.

[10] D. Enright, D. Nguyen, F. Gibou, and R. Fedkiw. Using the particle level set method and a second order accurate pressure boundary condition for free surface flows. In Proc. 4th ASMEJSME Joint Fluids Eng. Conf., number FEDSM2003-45144. ASME, 2003.

[11] F. Gibou, L. Chen, D. Nguyen, and S. Banerjee. A level set based sharp interface method for incompressible flows with phase change. J. Comput. Phys., 222:536-555, 2007. 
[12] F. Gibou and R. Fedkiw. A fourth order accurate discretization for the Laplace and heat equations on arbitrary domains, with applications to the Stefan problem. J. Comput. Phys., 202:577-601, 2005.

[13] F. Gibou, R. Fedkiw, R. Caflisch, and S. Osher. A level set approach for the numerical simulation of dendritic growth. J. Sci. Comput., 19:183-199, 2003.

[14] F. Gibou, R. Fedkiw, L.-T. Cheng, and M. Kang. A second-order-accurate symmetric discretization of the Poisson equation on irregular domains. J. Comput. Phys., 176:205-227, 2002.

[15] S. Gross and A. Reusken. An extended pressure finite element space for two-phase incompressible flows with surface tension. J. Comput. Phys., 224:40 - 58, 2007.

[16] Arthur Guittet, Mathieu Lepillez, Sebastien Tanguy, and Frederic Gibou. Solving elliptic problem with discontinuities on irregular domains - the Voronoi interface method. J. Comp. Phys. (submitted), 2015.

[17] A. Hansbo and P. Hansbo. An unfitted finite element method, based on nitsche's, method for elliptic interface problems. Comput. Methods Appl. Mech. Eng., 191:5537 - 5552, 2002.

[18] A. Hansbo and P. Hansbo. A finite element method for the simulation of strong and weak discontinuities in solid mechanics. Comput. Methods Appl. Mech. Eng., 193:3523 - 3540, 2004.

[19] A. Helgadottir and F. Gibou. A Poisson-Boltzmann solver on Irregular Domains with Neumann or Robin boundary conditions on Non-Graded Adaptive Grid. Journal of Computational Physics, 230 (10):3830-3848, 2011.

[20] T. Ho, Z. Li, S. Osher, and H. Zhao. A Hybrid Method for Moving Interface Problems with Application to the Hele-Shaw Flow. J. Comput. Phys., 134:236-252, 1997.

[21] S. Hou, W. Wang, and L. Wang. Numerical method for solving matrix coefficient elliptic equation with sharp-edged interfaces. J. Comput. Phys., 229:7162 - 7179, 2010.

[22] Z. Jomaa and C. Macaskill. The Shortley-Weller embedded finite-difference method for the 3D Poisson equation with mixed boundary conditions. J. Comput. Phys., 229:3675-3690, 2010. 
[23] M. Kang, R. Fedkiw, and X.-D. Liu. A boundary condition capturing method for multiphase incompressible flow. J. Sci. Comput., 15:323-360, 2000.

[24] B. Lamichhane and B. Wohlmuth. Mortar finite element method for elliptic problems with discontinuous coefficient. J. Numer. Anal., 22:549-576, 2002.

[25] R. LeVeque and Z. Li. The immersed interface method for elliptic equations with discontinuous coefficients and singular sources 31:1019-1044, 1994. SIAM J. Numer. Anal., 31:1019-1044, 1994.

[26] R. J. LeVeque and Z. Li. Immersed interface methods for stokes flow in elastic boundaries or surface tension. SIAM J. Sci. Comput., 18:1019 - 1044, 1997.

[27] X.D. Liu, R. Fedkiw, and M. Kang. A boundary condition capturing method for Poisson's equation on irregular domains. J. Comput. Phys., 160:151-178, 2000.

[28] F. Losasso, F. Gibou, and R. Fedkiw. Simulating water and smoke with an octree data structure. ACM Trans. Graph. (SIGGRAPH Proc.), pages 457-462, 2004.

[29] C. Min and F. Gibou. Geometric integration over irregular domains with application to level set methods. J. Comput. Phys., 226:1432-1443, 2007.

[30] Y-T. Ng, C. Min, and F. Gibou. An efficient fluid-solid coupling algorithm for single-phase flows. J. Comput. Phys., 228:8807-8829, 2009.

[31] D. Nguyen, R. Fedkiw, and M. Kang. A boundary condition capturing method for incompressible flame discontinuities. J. Comput. Phys., 172:71-98, 2001.

[32] M. Oevermann, C. Scharfenberg, and R. Klein. A sharp interface finite volume method for elliptic equations on Cartesian grids. J. Comput. Phys., 228:5184-5206, 2009.

[33] J. Papac, F. Gibou, and C. Ratsch. Efficient symmetric discretization for the Poisson, heat and Stefan-type problems with Robin boundary conditions. J. Comput. Phys., 229:875-889, 2010. 
[34] L. Parussini and V. Pediroda. Fictious Domain approach with hp-finite element approximation for incompressible fluid flow. J. Comput. Phys., 228:3891 - 3910, 2009.

[35] C. Peskin. Flow patterns around heart valves: A numerical method. J. Comput. Phys., 10:252$271,1972$.

[36] C. Peskin. Numerical analysis of blood flow in the heart. J. Comput. Phys., 25:220-252, 1977.

[37] C. Peskin. The immersed boundary method. Acta Numerica, 11:479-517, 2002.

[38] C. Peskin and B. Printz. Improved volume conservation in the computation of flows with immersed elastic boundaries. J. Comput. Phys., 105:146-154, 1993.

[39] J. W. Purvis and J. E. Burkhalter. Prediction of critical mach number for store configurations. AIAA J., 17:1170-1177, 1979.

[40] J. W. Purvis and J. E. Burkhalter. Prediction of critical mach number for store configurations. AIAA J., 17:1170-1177, 1979.

[41] Y. Saad. Iterative methods for sparse linear systems. PWS Publishing, 1996. New York, NY.

[42] J.-H. Song, P. Areias, and A. Belytschko. A method for dynamic crack and shear band propagation with phantom nodes. J. Numer. Methods Eng., 67:868 - 893, 2006.

[43] M. Sussman, P. Smereka, and S. Osher. A Level Set Approach for Computing Solutions to Incompressible Two-Phase Flow. Journal of Computational Physics, 114:146-159, 1994.

[44] Z. Tan, D. Le, Z. Li, K. Lim, and B. Khoo. An immersed interface method for solving incompressible viscous flows with piecewise constant viscosity across a moving elastic membrane. $J$. Comput. Phys., 227:9955 - 9983, 2008.

[45] A. Weigmann and K. Bube. The explicit-jump immersed interface method: finite difference method for pdes with piecewise smooth solutions. SIAM J. Sci. Comput., 37:827 - 862, 2000.

[46] Y. Zhou, S. Zhao, M. Feig, and G. Wei. High order mathced interterface and boundary method for elliptic equations with discontinuous coefficients and singular sources. J. Comput. Phys., $213: 1-30,2006$. 